TOPICAL REVIEW

\title{
Digital Fourier Microscopy for Soft Matter Dynamics
}

\author{
Fabio Giavazzi and Roberto Cerbino \\ Department of Medical Biotechnology and Translational Medicine, University of Milan, Italy \\ E-mail: roberto.cerbino@unimi.it
}

\begin{abstract}
Soft matter is studied with a large portfolio of methods. Light scattering and video microscopy are the most employed at optical wavelengths. Light scattering provides ensembleaveraged information on soft matter in the reciprocal space. The wave-vectors probed correspond to length scales ranging from a few nanometers to fractions of millimetre. Microscopy probes the sample directly in the real space, by offering a unique access to the local properties. However, optical resolution issues limit the access to length scales smaller than approximately $200 \mathrm{~nm}$. We describe recent work that bridges the gap between scattering and microscopy. Several apparently unrelated techniques are found to share a simple basic idea: the correlation properties of the sample can be characterised in the reciprocal space via spatial Fourier analysis of images collected in the real space. We describe the main features of such Digital Fourier Microscopy (DFM), by providing examples of several possible experimental implementations of it, some of which not yet realised in practice. We also provide an overview of experimental results obtained with DFM for the study of the dynamics of soft materials. Finally, we outline possible future developments of DFM that would ease its adoption as a standard laboratory method.
\end{abstract}

Keywords: microscopy, scattering, soft matter 


\section{Introduction}

Soft matter is a broad field encompassing a large variety of systems ubiquitous in our everyday life [1]. Typical examples are hair gel, paint, lipstick, mayonnaise, hair mousse, shampoo, and talc powder. Each of these complex fluids represents a class with some characteristic features. Polymers make up hair gels, paint is an example of colloid and lipstick may bear a liquid crystalline order. Mayonnaise is an emulsion, hair mousse is a foam, shampoos are complex surfactant solutions and talc powder is a granular material. All these systems share a sort of intermediate behaviour between liquids and solids and the interactions holding them together are of the order of $k_{B} T$. Such interactions are way weaker than those characterising liquids (typically a few tens of $k_{B} T$ ) but yet they determine and control the structure and dynamics of soft matter systems. In addition, they provide at the same time a certain degree of 'softness' i.e. a large responsiveness to external perturbations. Indeed, a rough estimate of the elastic modulus of soft materials is given by

$$
G \sim \frac{k_{B} T}{a^{3}}
$$

where $a$ is some typical characteristic length of the soft material, whose order of magnitude lies typically in the range $[10 \mathrm{~nm}, 100 \mu \mathrm{m}]$. This gives rise to an elastic modulus of the order $G \sim[n P a, k P a]$, which is way smaller than the ones characterising typical solids $(G \sim 10-1000$ $G P a$ ), accounting for the softness of these materials. The study of materials with length scales varying in such a wide range requires the combination of several experimental methods, including microscopy and scattering with radiation of different kind and wavelength, the most relevant being light, neutrons, and x-rays $[2,3,4,5,6,7,8,9,10,11]$.

Even with this large availability of tools, optical methods remain of paramount importance both in real and reciprocal space. In the past, light scattering has been the traditional choice for quantitative studies of the structural and dynamical properties of soft materials [10], even though in the last years there has been a steadily growing use of microscopy techniques [5]. Historically, scattering and microscopy have been long thought of as very distinct tools. Light scattering can access large regions of the sample probing time scales down to the nanosecond. In addition, it benefits from the possibility of ensemble averages over a large number of scatterers and it provides easy access to three dimensional statistical properties of the sample. On the other hand, microscopy provides an invaluable map of the sample in the real space and allows an easier probe of heterogeneous samples, in particular when discriminating sub-populations of differently-featured entities is important.

Technological advances leading to better computer performances and improved chargecoupled device (CCD) or complementary-metal-oxide-semiconductor (CMOS) cameras - in terms of resolution, acquisition speed and noise properties - have recently enabled the practical possibility of using imaging devices in scattering experiments. Initially, cameras have been successfully employed in far-field scattering experiments, both for static [12] and dynamic [13] applications, for which they immediately proved to be a reasonable alternative to other detectors. The comparative slowness and increased noise of cameras with respect to photomultiplier tubes and avalanche photodiodes turned out to be compensated by the possibility of performing multi-speckle detection on different camera pixels, with obvious advantages for nonergodic samples.

More recently M. Giglio and coworkers $[14,15]$ dropped the idea of having the camera in the far field of the scattering sample and collected images in its close proximity (the so called near field or deep Fresnel regime [16]), but still at some minimum distance from it to ensure the presence of speckles [17]. In the deep Fresnel regime, the speckle size does not change with the 
distance from the sample as a consequence of the conservation of the angular spectrum of light upon propagation $[18,19,20]$. In addition, under the hypothesis that the scattering object is weak, the image intensity is de facto a coherent hologram of the sample, with the real part of the scattered field encoded in the image intensity. As a consequence, the Fourier power spectrum of the near field images provides directly the scattering intensity $I(Q)$ as a function of the scattering wave-vector $Q$, multiplied by a transfer function that accounts for the details of the experimental setup [16]. Whenever the transfer function can be neglected or independently measured it is thus possible to perform static light scattering (SLS) experiments at small scattering angles by studying images collected in the deep Fresnel regime. This heterodyne near field scattering (HNFS), as named by its proposers, is strongly connected to the so called quantitative shadowgraphy [21, 22], a technique that was developed a few years before for the quantitative study of instabilities and non-equilibrium fluctuations in fluids. The strong link between the two methods was realised only later, when it was understood that shadowgraphy and HNFS, rather then being two distinct methods, are actually the limit of the same technique for small and large distances (or wave-vectors), respectively [23]. The transfer function of HNFS is only affected by the numerical aperture of the collection optics and by the coherence of light, whereas shadowgraphy suffers from additional oscillations due to the Talbot effect [16].

A few years later, both methods were indeed simultaneously extended to extract also the system dynamics $[24,25,26,27]$ from the analysis of images acquired at different times. This analysis provides information similar to that obtained with Dynamic Light Scattering (DLS) [4], once again in the small-angle regime. Measuring the dynamics is easier than measuring $I(Q)$, because it is not necessary to determine the time-independent transfer function. The method works also with hard $\mathrm{x}$-rays where the dynamics of a colloidal suspension was determined by Fourier analysis of deep Fresnel images [28], without need of pinholes or other devices usually employed for increasing the coherence of synchrotron radiation. Another step forward was accomplished in Ref. [29], where a commercial microscope equipped with a halogen lamp was used to collect images in the mid-plane of a thin capillary containing a dilute colloidal suspension. The resulting Differential Dynamic Microscopy (DDM), in contrast with shadowgraphy and HNFS, does not use any specialised source or setup and operates inside the sample, where in most cases no speckles can be even observed. The results in Ref. [29] proved indeed that DDM was capable of providing high-quality studies of the dynamics both for large particles, whose motion could be easily followed with particle tracking, and for sub-diffraction scatterers, which can not be individually resolved with visible light.

Closely connected to all the above methods is also the so called Fourier Transform Light Scattering (FTLS) in which, under some hypothesis about the sample, the optical phase and amplitude of a coherent image field are quantified and propagated numerically to the scattering plane for performing both SLS and DLS [30].

Related but independent work was made in the biophysical community where, starting from ideas closer to fluorescence correlation spectroscopy (FCS) [31] than to DLS, it was shown that the typical FCS real space analysis of number fluctuations in a small volume [32] could be replaced by a dynamic analysis of Fourier transformed microscope images with the aim of extracting simultaneous information on the sample dynamics at various length scales [33]. The principle of the method differs from the one of scattering based techniques in that the intensity signal is due to fluorescence which notoriously leads to incoherent imaging.

At first, the fact that incoherent, coherent, or partially coherent methods based on either scattering or fluorescence give the same result may seem accidental. However, it is possible to show - and we will do it in more detail in this review article - that under rather general but still 
somehow restrictive conditions [34], the analysis of microscope images in the Fourier space - from here on Digital Fourier Microscopy (DFM) - of a sample can provide quantitative information about its structure and dynamics similar to the one obtained in SLS and DLS experiments. The reader should be aware that the term microscopy is used here in a very broad sense because it includes many situations where, due to defocusing [35], speckles [17] or other effects the image of the sample does not seem to bear any resemblance with the sample itself.

It is worth concluding this Introduction by mentioning that DFM is not the only approach that exploits imaging to characterise soft matter samples. Indeed, in the last few years other nonFourier methods have been proposed, such as for instance Photon Correlation Imaging [36] or Echo Speckle Imaging [37], whose focus is the space-resolved characterisation of soft matter dynamics with simultaneous high temporal and spatial resolution. The reader interested in a review of such methods in connection to the ones treated here should refer to Ref. [16].

\section{Theory of Digital Fourier Microscopy}

An equivalent description of the dynamics of soft matter samples can be either given in the real space or in the reciprocal space. The real space approach is apparently more convenient because it is naturally and more directly related to our everyday experience. If we focus for instance on a colloidal suspension of particles, the success of a real space approach depends crucially on the fact that the particles can be individually and unambiguously tracked for the whole duration of the experiment. This requirement is very difficult to satisfy for particles that are much smaller than the wavelength of light because their contrast decreases quite rapidly with their size (for instance the scattering cross section scales with the square of the particle volume) and also because they generally move fast. In addition, when the particles' concentration gets larger, it becomes increasingly difficult to perform an accurate tracking. If the sample is not made of particles but is instead composed of small molecules, tracking with visible light becomes practically impossible. In all cases for which tracking is possible, one can extract various quantities characterising the motion of individual particles [38] with obvious advantages over far-field scattering techniques in all those cases where the sample is polydisperse or inhomogeneous. However, whenever the dynamics of the system under study is described in real space by linear partial differential equations, the reciprocal space description allows transforming the partial differential equations into algebraic equations and gives direct access to the eigenmodes of the system [2,4]. This property increases the appeal of the reciprocal space approach also when there is the possibility of tracking the particles in real space.

In this review article we describe a family of apparently unrelated techniques that have been developed during the last years and that are characterised by the same basic idea: by collecting a stack of microscope images of the sample and performing a temporal analysis of the spatially Fourier transformed images, information can be extracted that is equivalent to the one obtained in static and dynamic light scattering experiments. This is the principle of DFM, as it will be described in more detail in the following paragraphs.

\subsection{The sample}

For definiteness we assume that the spatio-temporal behaviour of the sample can be described by a time-dependent scalar field $c(\mathbf{X}, t)$, where $\mathbf{X}$ is a three-dimensional (3D) space coordinate. Such scalar field $c(\mathbf{X}, t)$ can be thought of as a generalised density. This description is suitable for continuous variables such as for instance the concentration of one component in a binary fluid 
or a given vectorial component of the director in a fluctuating nematic liquid crystal. The same formalism can be also used to describe a set of $N$ discrete particles in a volume $V$ for which $c(\mathbf{X}, t)$ can be identified with the particle number density

$$
c(\mathbf{X}, t)=\sum_{n=1, . ., N} \delta\left(\mathbf{X}-\mathbf{X}_{n}(t)\right) .
$$

For more complex cases where the description of the system requires a vector field (e.g. velocity), a tensor field (e.g. dielectric tensor or stress tensor) or more than one scalar field (e.g. concentrations in a multicomponent system) the generalisation of such description should be quite straightforward.

Crucial features of the space-time behaviour for a translationally invariant system are captured by the space-time correlation function

$$
C(\Delta \mathbf{X}, \Delta t)=\langle c(\mathbf{X}+\Delta \mathbf{X}, t+\Delta t) c(\mathbf{X}, t)\rangle
$$

or equivalently by the van Hove correlation function

$$
G(\Delta \mathbf{X}, \Delta t)=\frac{1}{\langle c\rangle}\langle c(\mathbf{X}+\Delta \mathbf{X}, t+\Delta t) c(\mathbf{X}, t)\rangle
$$

where the symbol $\langle\square\rangle$ is used here either as a temporal average or as ensemble average under the assumption that the system of interest is ergodic [2,4]. In general, the van Hove correlation function can be written as the sum of a "self" part $G_{s}(\Delta \mathbf{X}, \Delta t)$ and a "distinct" one $G_{d}(\Delta \mathbf{X}, \Delta t)$, where the self part describes the probability (density) for a particle to make a displacement $\Delta \mathbf{X}$ in the time interval $\Delta t$ and the distinct part accounts for interparticle correlations. If a system of statistically independent particles is considered $G(\Delta \mathbf{X}, \Delta t)=G_{s}(\Delta \mathbf{X}, \Delta t)$.

In the literature several other functions that describe the correlation properties of the sample in the reciprocal space have been introduced, especially in connection with static and dynamic scattering experiments. If we indicate the 3D Fourier transform operation with the symbol $\hat{\square}$ and we define $N=\int d^{3} \mathbf{X} c(\mathbf{X}, t)$, we can introduce the intermediate scattering function

$$
F(\mathbf{Q}, \Delta t)=\frac{1}{N}\left\langle\hat{c}(\mathbf{Q}, t+\Delta t) \hat{c}^{*}(\mathbf{Q}, t)\right\rangle=\hat{G}(\mathbf{Q}, \Delta t)
$$

or its normalised version

$$
f(\mathbf{Q}, \Delta t)=\frac{F(\mathbf{Q}, \Delta t)}{F(\mathbf{Q}, 0)} .
$$

Another function often found in the literature is the structure function

$$
D(\mathbf{Q}, \Delta t)=\frac{1}{N}\left\langle|\hat{c}(\mathbf{Q}, t+\Delta t)-\hat{c}(\mathbf{Q}, t)|^{2}\right\rangle=2\{\hat{G}(\mathbf{Q}, 0)-\Re[\hat{G}(\mathbf{Q}, \Delta t)]\}
$$

or its normalised version

$$
d(\mathbf{Q}, \Delta t)=\frac{D(\mathbf{Q}, \Delta t)}{D(\mathbf{Q},+\infty)}=1-\Re[f(\mathbf{Q}, \Delta t)]
$$

where $\Re[a]$ denotes the real part of $a$. In terms of the above defined intermediate scattering function the well known dynamic structure factor

$$
S(\mathbf{Q}, \omega)=\frac{1}{2 \pi} \int d t e^{-j \omega t} F(\mathbf{Q}, \Delta t)
$$




\begin{tabular}{|c|c|c|c|c|}
\hline & Brownian & Uniform velocity $\mathbf{V}_{\mathbf{0}}$ & \multirow{2}{*}{\multicolumn{2}{|c|}{ Velocity distribution $P(V)$}} \\
\hline & $\Gamma(Q)=D_{0} Q^{2}$ & $\Gamma(\mathbf{Q})=\mathbf{Q} \cdot \mathbf{V}_{0}$ & & \\
\hline$G(\Delta \mathbf{X}, \Delta t)$ & $\frac{\exp \left(-\frac{|\Delta X|^{2}}{4 D_{0} t}\right)}{(4 \pi D \Delta t)^{\frac{3}{2}}}$ & $\delta\left(\Delta \mathbf{X}-\mathbf{V}_{0} \Delta t\right)$ & \multicolumn{2}{|c|}{$\frac{1}{\Delta t} P(|\Delta X| / \Delta t)$} \\
\hline$F(\mathbf{Q}, \Delta t)$ & $e^{-\Gamma(Q) \Delta t}$ & $e^{j \Gamma(Q) \Delta t}$ & $4 \pi \int_{0}^{\infty} d V V^{2} P(V)$ & $\frac{\sin (Q V \Delta t)}{Q V \Delta t}$ \\
\hline$D(\mathbf{Q}, \Delta t)$ & $2\left[1-e^{-\Gamma(Q) \Delta t}\right]$ & $2[1-\cos (\Gamma(\mathbf{Q}) \Delta t)]$ & \multicolumn{2}{|c|}{$2[1-F(\mathbf{Q}, \Delta t)]$} \\
\hline$S(\mathbf{Q}, \omega)$ & $\frac{1}{\pi} \frac{\Gamma(Q)}{\Gamma^{2}(Q)+\omega^{2}}$ & $\delta(\omega-\Gamma(\mathbf{Q}))$ & \multicolumn{2}{|c|}{$2 \pi \int_{0}^{\omega / q} d V V^{2} P(V)$} \\
\hline
\end{tabular}

Table 1. Van Hove correlation function $G(\Delta \mathbf{X}, \Delta t)$, intermediate scattering function $F(\mathbf{Q}, \Delta t)$, structure function $D(\mathbf{Q}, \Delta t)$ and dynamic structure factor $S(\mathbf{Q}, \omega)$ calculated for three simple systems of particles: independent particles in Brownian motion with diffusion coefficient $D_{0}$; particles translating with uniform velocity $\mathbf{V}_{\mathbf{0}}$; particles moving with an isotropic velocity distribution $P(V)$.

and static structure factor

$$
S(\mathbf{Q})=\int S(\mathbf{Q}, \omega) d \omega=F(\mathbf{Q}, 0)
$$

are defined.

In Table 1 we report the relevant expressions for some of the above-defined correlation functions relative to three paradigmatic dynamic systems. The three columns describe a system of independent particles of arbitrary size in Brownian motion with diffusion coefficient $D_{0}$ (first column), in uniform motion with velocity $\mathbf{V}_{0}$ (second column) and in motion with an isotropic velocity distribution $P(V)$ (third column).

\subsection{Scattering}

Light scattering is the ideal probe of the sample correlation properties in the reciprocal space. In light scattering experiments a light beam (ideally a plane wave with wave-vector $\vec{k}_{i}$ ) is impinging on a sample of interest, considered here for simplicity non-magnetic, non-conducting and nonabsorbing. In general, as the plane wave progresses through the sample, both the amplitude and the phase of its electric field change as a consequence of local spatio-temporal variations $\delta \epsilon(\mathbf{X}, t)$ of the sample dielectric constant $\epsilon(\mathbf{X}, t)=\epsilon_{0}+\delta \epsilon(\mathbf{X}, t)$. Here $\epsilon_{0}$ is the average dielectric constant and $n=\sqrt{\epsilon_{0}}$ the corresponding refractive index. For a weakly scattering object the first-order Born approximation is usually made, according to which the illuminating beam propagates unchanged within the sample and is diffracted $\grave{a}$ la Bragg by three-dimensional gratings originated by the Fourier components $\delta \hat{\epsilon}(\mathbf{Q}, t)=\int \delta \epsilon(\mathbf{X}, t) e^{i \mathbf{Q} \cdot \mathbf{X}} d^{\mathbf{3}} \mathbf{X}$ of the refractive index variations.

For each scattering wave vector $\mathbf{Q}$ and far away from the sample (Fig. 1a), the scattered light emerges as a plane wave traveling with wave-vector $\mathbf{k}_{s}$ such that $\mathbf{Q}=\mathbf{k}_{i}-\mathbf{k}_{s}$. For elastic scattering processes $\left(k_{i}=k_{s} \doteq k\right)$, the wave-vector $\mathbf{Q}$ is linked to a well prescribed scattering angle $\theta$, as measured with respect to the propagation direction of the transmitted beam (Fig. 1b) and one has

$$
Q=2 k \sin \left(\frac{\theta}{2}\right)
$$

For each angle $\theta$, the amplitude of the electric field of the scattered plane wave is directly proportional to the Fourier component $\delta \hat{\epsilon}(\mathbf{Q}, t)[39,40]$. However, at optical frequencies detectors 




Figure 1. (a) Sketch of a typical far field scattering experiment with the detector placed far away from the sample. (b) Wave-vectors involved in the scattering process with $\mathbf{Q}=\left(\mathbf{q}, q_{z}\right)$. (c) Sketch of a typical DFM experiment. Light impinging on the sample is scattered at various angles and is collected by the objective lens. 2D microscope images of the sample are Fourier analysed and information equivalent to a traditional far-field scattering experiment (a) is recovered. A generic scattered ray (wave propagating) with polar angle $\theta$ and azimuthal angle $\phi$ (dashed line), which corresponds to the point $(\theta, \phi)$ in the far-field scattering pattern (a), is collected by the lens in a DFM experiment (c) and contributes to the image. The contribution of each scattered ray (wave) can be isolated by means of a two dimensional Fourier analysis, which is based on the two-dimensional projection $\mathbf{q}$ of the wave vector $\mathbf{Q}$ transferred during the scattering process (b).

are sensitive to the light intensity

$$
I(\mathbf{Q}, \mathrm{t}) \propto|\delta \hat{\epsilon}(\mathbf{Q}, t)|^{2}
$$

and thus only the Fourier power spectrum $|\delta \hat{\epsilon}(\mathbf{Q}, t)|^{2}$ of the dielectric constant variations can be probed with scattering experiments.

Usually, in static light scattering (SLS) experiments the time-averaged intensity

$$
I(\mathbf{Q})=\langle I(\mathbf{Q}, t)\rangle_{t}
$$

is studied as a function of the scattering wave vector $\mathbf{Q}$, whereas dynafmic light scattering (DLS) probes, for one or more scattering wave vectors $\mathbf{Q}$, the temporal autocorrelation of the scattering intensity

$$
G_{I}(\mathbf{Q}, \Delta t)=\langle I(\mathbf{Q}, t+\Delta t) I(\mathbf{Q}, t)\rangle_{t}
$$




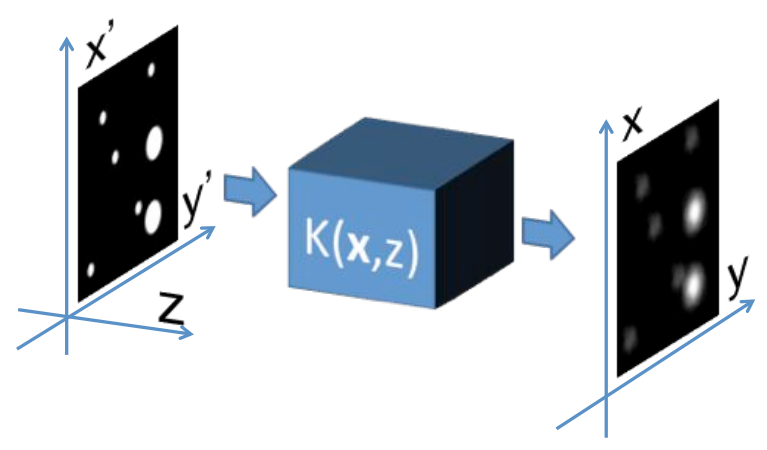

Figure 2. Pictorial representation of a linear space invariant imaging system. Each thin slice of a $3 \mathrm{D}$ sample is imaged onto the $(x, y)$ plane as a convolution integral of the density distribution $c(\mathbf{x}, z, t)$ with the z-dependent kernel $K(\mathbf{x}, z)$. All such contributions add up to produce the image intensity $i(x, y)$, as described by Eq.16.

or its normalised version

$$
g_{I}(\mathbf{Q}, \Delta t)=\frac{\langle I(\mathbf{Q}, t+\Delta t) I(\mathbf{Q}, t)\rangle_{t}}{\langle I(\mathbf{Q}, t)\rangle_{t}^{2}} .
$$

SLS and DLS experiments can thus provide information about the spatio-temporal features of the variations of the dielectric constant $\delta \epsilon(\mathbf{X}, t)$ - and in turn of the density $c(\mathbf{X}, t)$ - within the sample.

\subsection{Imaging}

The basic idea of DFM can be captured by relying on the intriguing analogy presented in Fig. 1 . In DFM one collects microscopy images of the sample, instead of measuring the scattering intensity in the far field. Independent on the imaging being based on scattering or fluorescence or on some other mechanism, we will show that the analysis of the correlation properties of the images in the reciprocal space provides information about the correlation properties of the sample analogous to that extracted by light scattering. This analogy is based on some very general assumptions about the imaging process that are discussed below.

2.3.1. Linear space invariant (LSI) imaging of $3 D$ samples It has been demonstrated in Ref. [34] that DFM works whenever the imaging process is such that a LSI relation holds between the timedependent image intensity distribution $i(\mathbf{x}, t)$ and the sample density $c(\mathbf{x}, z, t)$ i.e. when the image can be written as a convolution integral of the form:

$$
i(\mathbf{x}, t)=i_{0}+\int d z \int d^{2} \mathbf{x}^{\prime} K\left(\mathbf{x}-\mathbf{x}^{\prime}, z\right) c(\mathbf{x}, z, t)
$$

where $K(\mathbf{x}, z)$ is a generalised 3D point-spread function (PSF), $i_{0}$ is a nearly uniform background contribution which is assumed to be independent of the sample and where $\mathbf{X}=(\mathbf{x}, z)$. Eq. 16 should be understood as a sufficient but not necessary condition for the validity of the DFM approach and until now it has been satisfied, to the best of our knowledge, for all the existing DFM methods. Starting from Eq. 16 we obtain the 2D Fourier transform of the image intensity

$$
\hat{i}(\mathbf{q}, t)=i_{0} \delta(\mathbf{q})+\int d q_{z} \hat{K}\left(\mathbf{q}, q_{z}\right) \hat{c}\left(\mathbf{q}, q_{z}, t\right)
$$



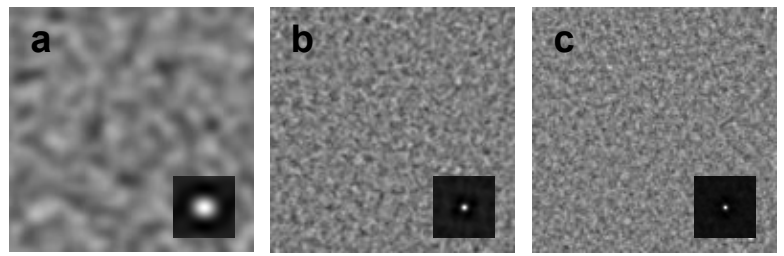

Figure 3. Bright field images of an aqueous suspension (volume fraction $\phi=0.01$ ) of 100 $\mathrm{nm}$ polystyrene nanoparticles confined in a $100 \mu \mathrm{m}$ thick capillary. In order to isolate the fluctuating part, a background contribution obtained by averaging over 1000 independent images, was subtracted to each image. In each panel, an image obtained with magnification $M$ and numerical aperture $N A$ is shown: a) $M=5, N A=0.15$, b) $M=20, N A=0.45$, c) $M=40$, $N A=0.6$. To compare the size of the speckles obtained with different magnification, panels a) and b) were additionally magnified by a factor eight and two, respectively, so that the side of each panel corresponds to about $75 \mu \mathrm{m}$ in real space. As a consequence of the increased numerical aperture of the objective, the speckle size decreases markedly from a) to c), which can be also appreciated in the insets, where the two-dimensional autocorrelation of the corresponding images are shown.

where $\hat{K}\left(\mathbf{q}, q_{z}\right)$ is a the 3D generalised transfer function, i.e. the 3D Fourier transform of the generalised PSF and where $\mathbf{Q}=\left(\mathbf{q}, q_{z}\right)$ (Figure 1c). In Eq. 17 we have used the same symbol $\hat{\square}$ for both the 2D and the 3D Fourier transforms of real space quantities. This choice will be pursued also in the following and the relevant dimensionality can be inferred by inspection of the argument: $\mathbf{Q}=\left(\mathbf{q}, q_{z}\right)$ is used for $3 \mathrm{D}$ functions and $\mathbf{q}$ for $2 \mathrm{D}$ ones.

In Eq. 16 we have assumed that the magnification $M$ for the optical system is unit or, equivalently, that the image coordinate $\mathbf{x}$ has been rescaled in order to account for the system magnification: $\mathbf{x} \rightarrow \mathbf{x} / M$. It is also worth pointing out that, in general, the validity of Eqs. 16 and 17 is not compromised by the presence of optical aberrations. In fact, many (even though not all) deviations from an ideal imaging process usually considered as aberrations (e.g. defocusing, astigmatism, spherical aberration) preserve the linear-space invariant relation between the object and image defined by Eq. 16. Their effect can be thus incorporated in the generalised PSF $K(\mathbf{x}, z)$.

In contrast with several forms of modern microscopy that are interested in the morphological details of small objects, DFM experiments are not based on the image being a good reproduction of the object from an aesthetical point of view. Indeed, in many cases for which the DFM approach allows an accurate measurement of the dynamics of the sample, it is absolutely impossible to retrieve the positions or even the shape or size of the original constituents from the intensity distribution of the images. A striking example is provided in Figure 3, where three bright-field images of a concentrated suspension of sub-micron particles obtained with three different numerical apertures $(N A)$ are shown, after being rescaled to account for the different magnification of the objective. In this case the sample, which is made of colloidal particles of $100 \mathrm{~nm}$ (diameter) suspended in water, is prepared at a volume fraction that causes the typical inter-particle distance to be much shorter than the characteristic lateral dimension of the PSF. As a consequence, the intensity in each point of the image is determined by the superposition of the contributions from a large number of particles, which originates images with a speckled appearance, the speckle size being determined only by the width of the PSF. Such inability of resolving the individual particles does not affect the performances of DFM, as it will be shown in the following (see for instance Fig. 8). 
2.3.2. Dynamic imaging of $3 D$ samples Eq. 16 provides the link between the image intensity and the generalised density. With Eq. 16 in mind it is thus possible to define, in analogy with Eqs. 5 and 9, the image intermediate scattering function

$$
F_{i}(\mathbf{q}, \Delta t) \doteq\left\langle\hat{i}(\mathbf{q}, t+\Delta t) \hat{i}^{*}(\mathbf{q}, t)\right\rangle
$$

and its normalised version

$$
f_{i}(\mathbf{q}, \Delta t) \doteq \frac{F_{i}(\mathbf{q}, \Delta t)}{F_{i}(\mathbf{q}, 0)}
$$

Analogous definitions can be given for the image structure function $D_{i}(\mathbf{q}, \Delta t) \dot{=}$ $\left\langle|\hat{i}(\mathbf{q}, t+\Delta t)-\hat{i}(\mathbf{q}, t)|^{2}\right\rangle$ and its normalised version $d_{i}(\mathbf{q}, \Delta t) \doteq \frac{D_{i}(\mathbf{q}, \Delta t)}{D_{i}(\mathbf{q}, 0)}$, for the image dynamic structure factor $S_{i}(\mathbf{q}, \omega) \doteq \int d t e^{-j \omega t} F_{i}(\mathbf{q}, t)$ and for the image static structure factor $S_{i}(\mathbf{q})=$ $\int S_{i}(\mathbf{q}, \omega) d \omega=F_{i}(\mathbf{q}, 0)$.

We stress that all the characteristic functions defined on the images depend on the $2 \mathrm{D}$ wavevector q, whereas the corresponding functions characterising the density depend on the 3D wavevector Q. Substitution of Eq. 16 in Eqs. 18 and 19 provides

$$
\begin{aligned}
& F_{i}(\mathbf{q}, \Delta t)=N \int d q_{z}\left|\hat{K}\left(\mathbf{q}, q_{z}\right)\right|^{2} F\left(\mathbf{q}, q_{z}, \Delta t\right) \\
& f_{i}(\mathbf{q}, \Delta t)=\frac{\int d q_{z}\left|\hat{K}\left(\mathbf{q}, q_{z}\right)\right|^{2} F\left(\mathbf{q}, q_{z}, \Delta t\right)}{\int d q_{z}\left|\hat{K}\left(\mathbf{q}, q_{z}\right)\right|^{2} F\left(\mathbf{q}, q_{z}, 0\right)} .
\end{aligned}
$$

It appears that in general, as a consequence of the 3D nature of the imaging process, there is not a direct proportionality between $f_{i}(\mathbf{q}, \Delta t)$ and $f(\mathbf{q}, \Delta t)$ and the dynamics at a given wave-vector $\mathbf{q}$ is potentially affected also by the axial dynamics associated with $q_{z}$.

$2 D$ sample It is interesting to notice that the proportionality between $f_{i}(\mathbf{q}, \Delta t)$ and $f(\mathbf{q}, \Delta t)$ is recovered for a 2D sample for which Eqs. 20 and 21 simplify to

$$
\begin{aligned}
& F_{i}(\mathbf{q}, \Delta t)=N|\hat{K}(\mathbf{q})|^{2} F(\mathbf{q}, 0, \Delta t) \\
& f_{i}(\mathbf{q}, \Delta t)=f(\mathbf{q}, 0, \Delta t) .
\end{aligned}
$$

Eq. 23 implies that the image normalised structure function actually coincides with the normalised structure function of the sample density, which is the ideal working condition for DFM, in which the details of the imaging process become irrelevant.

Can the axial dynamics be tamed? Luckily, as shown in Ref. [34], in many cases of practical relevance it is possible to recover the proportionality between the image and the sample intermediate scattering functions also for 3D samples, at least in a given wave-vector range. From Eq. 20 it appears that the image intermediate scattering function at a given $\mathbf{q}$ is obtained as a weighted average of contributions from a whole interval of $3 \mathrm{D}$ wave-vectors $\mathbf{Q}=\left(\mathbf{q}, q_{z}\right)$, having the same transverse component $\mathbf{q}$ and different $q_{z}$. The range of axial wave-vectors $q_{z}$ giving a significant 
contribution can be estimated by evaluating the axial width of the convolution kernel $\left|\hat{K}\left(\mathbf{q}, q_{z}\right)\right|^{2}$ defined by

$$
\Delta q(\mathbf{q}) \doteq \sqrt{\frac{\int d q_{z} q_{z}^{2}\left|\hat{K}\left(\mathbf{q}, q_{z}\right)\right|^{2}}{\int d q_{z}\left|\hat{K}\left(\mathbf{q}, q_{z}\right)\right|^{2}}} .
$$

If the transverse dynamics associated to the $q_{z}$-values in the interval $\left|q_{z}\right| \lesssim \Delta q(\mathbf{q})$ is significantly slower than the corresponding transverse dynamics (associated with q), i.e if

$$
F\left(\mathbf{q}, q_{z}, \Delta t\right) \simeq F(\mathbf{q}, 0, \Delta t)
$$

then the validity of Eqs. 22 and 23 is recovered, at least approximately, even in this case. Since in most cases the characteristic correlation time is a decreasing function of $q$, a criterion for the validity of Eqs. 22 and 23 can be given in the form

$$
|F(\mathbf{q}, \Delta q, \Delta t) / F(\mathbf{q}, 0, \Delta t)-1| \ll 1 .
$$

It has been shown in Ref. [34] that for bright-field DFM of freely diffusing particles, the condition for image formation is more severe than the inequality in Eq. 26, which implies that for all those $\mathbf{q}$ for which there is signal in the images, the dynamics can be safely assessed. In the next section several examples of generalised PSF are given for which a $2 \mathrm{D}$ image processing provides information about the 3D sample dynamics. A notable case for which the effect of the axial dynamics becomes evident in DFM experiments also for freely diffusing particles is represented by the confocal microscope [41].

\subsection{Experimental realisations of DFM}

Two representative experimental setups suitable for performing DFM are reported in Figs. 4 and 5, where a typical bench-top "microscope" and a commercial one are sketched.

An optical arrangement very similar to the one shown in Fig. 4 is shared by all the seminal experiments in which DFM was first attempted. A typical signature is the of use an illumination with high spatial coherence (ideally a single transverse mode) from a narrow-banded light source (usually a fibre-coupled led or laser). This kind of set-up is particularly flexible: for example it is quite straightforward to insert suitable filters (items a-d in position 6 in Fig. 4) in the back focal plane of the objective lens and rapidly switch from bright-field [14] to phase contrast, dark-field [15] or Schlieren [42] detection . Moreover, in absence of mechanical constraints, it is quite simple to translate the camera along the optical axis even very far away from the plane conjugate with the sample, as it is usually done in shadowgraphy [43].

Although much less flexible, an unmodified, commercial microscope (Figure 5), is typically much easier to handle than a custom, bench-top instrument. Moreover, it displays a number of very interesting features even from a purely optical point of view: tuneable spatial and temporal coherence of the illumination beam; calibrated and aberrations-corrected objectives in a wide range of magnifications and numerical apertures; very stable, pre-aligned optical path; simplified adjustment procedures, just to mention a few examples. For this set-up it is also possible to switch from bright-field mode to advanced imaging modalities such as for instance phase-contrast or dark-field by placing pairs of suitably shaped conjugated apertures in the front focal plane of 


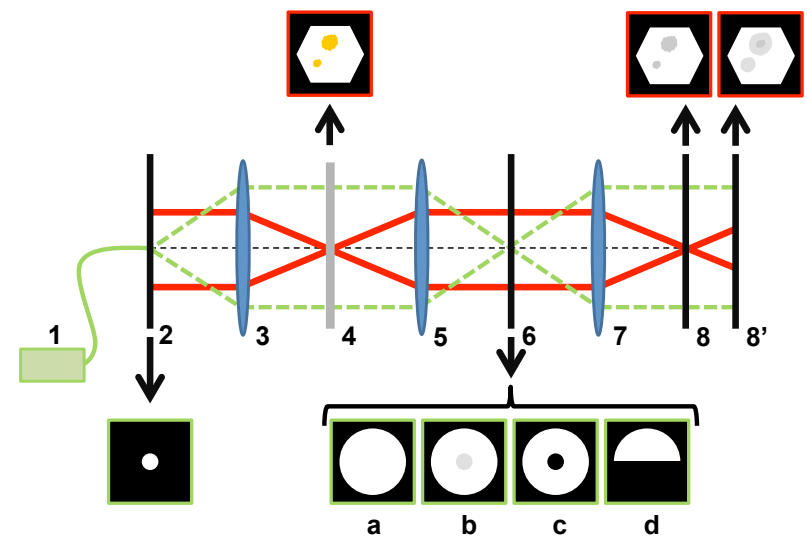

Figure 4. Sketch of a typical bench-top microscope. The red continuous lines indicate the image forming light path and the green dashed lines the illumination light path. 1-fibre-coupled laser or led source; 2-fibre tip; 3-collimation lens; 4-object plane (sample), 5-objective lens, 6-objective back focal plane where the following objects can be placed: (a) nothing for bright-field (b) phase retarding plate in the focal point for phase contrast (c) beam stop in the focal point for darkfield (d) knife-edge for Schlieren; 7-relay lens; 8-image plane (camera sensor); 8'-defocused image plane.

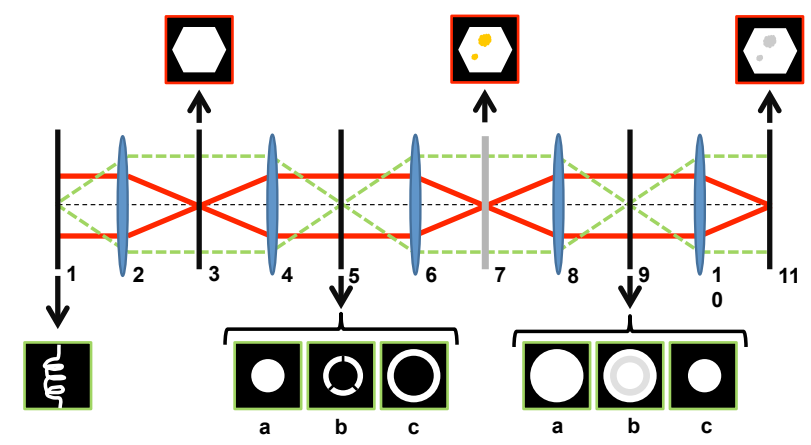

Figure 5. Sketch of a typical commercial microscope in Koehler configuration. The red continuous lines indicate the image forming light path and the green dashed lines the illumination light path. 1-incoherent light source (lamp filament); 2-collector lens; 3-field diaphragm; 4-field lens; 5-condenser lens front focal plane where the following objects can be placed: (a) aperture diaphragm for bright-field (b) phase contrast ring (c) dark-field light stop; 6-condenser lens; 7object plane (sample); 8-objective lens; 9-objective back focal plane where the following objects must be placed, according to the choice made in 5: (a) nothing for bright field (b) phase retarding ring for phase contrast (c) nothing for dark-field (low NA objective); 10-relay lens; 11-image plane (camera sensor). 
the condenser (items a-c in position 5 in Fig. 5) and in the back focal plane of the objective (items a-c in position 9 in Fig. 5).

In the remaining part of this section several examples of microscopy experiments are presented, all compatible with both the described set-ups and fulfilling the requirements for the validity of Eq. 16.

2.4.1. Scattering-based microscopy In microscopy observations of weakly scattering or weakly absorbing objects the optical signal associated to the fluctuating density $c$ is due to the induced variation in the complex refractive index of the sample. If $E_{0}$ is the scalar field incident on the sample and $E_{t}$ is the transmitted field we can write the total field after the sample as $E=E_{t}+E_{s}$, where $E_{s}$ is the scattered field. The object is weak if $\left|E_{s}\right| \ll\left|E_{0}\right|$ i.e. if $I_{t} \simeq I_{0}$, where $I_{t, 0}=\left|E_{t, 0}\right|^{2}$. This is the so called heterodyne regime where, momentarily neglecting the effect of the collection optics, the intensity distribution in the image plane is given by

$$
i(\mathbf{x}, t) \propto|E(\mathbf{x}, t)|^{2} \simeq\left|E_{t}\right|^{2}+2 \Re\left(E_{t}^{*} E_{s}\right)
$$

It is obvious that the heterodyne condition is not compatible with methods that get rid of the transmitted beam such as dark field microscopy or polarised microscopy with crossed polarisers. In those cases, the $I_{s}=\left|E_{s}\right|^{2}$ term, neglected in Eq. 27, becomes the only contribution to the image intensity (see paragraph 2.4.3 for a brief discussion of such cases).

Bright-field microscopy Immediately after the sample, the scattered field can be written as $E_{s}=\left(\sigma_{A}+j \sigma_{P}\right) E_{0}$. Here $\sigma_{A}$ and $\sigma_{P}$ represent the phase and amplitude modulations introduced by the sample on the impinging wave front, respectively. The transmitted field coincides with the incident field $E_{t} \simeq E_{0}$. For a $2 \mathrm{D}$ sample, the phase and amplitude modulations of the field are actually produced by inhomogeneities of the real and imaginary part of the sample refractive index, respectively. The image intensity turns out to be proportional to the amplitude modulation $\sigma_{A}$ introduced by the sample $i \propto I_{0}\left(1+2 \sigma_{A}\right)$. When both the 3D structure of the sample and defocusing are taken into account, the relationship becomes in general more complicated and phase modulations become also visible, which is actually the same principle of shadowgraphy. A detailed model of the PSF of a bright field microscope, derived following Refs. [45, 44], can be found in Ref. [34] where an analytical expression for the transfer function of a bright-field microscope is obtained in the form

$$
\hat{K}\left(\mathbf{q}, q_{z}\right)=T\left(\mathbf{q}, q_{z}\right) \pm T\left(\mathbf{q},-q_{z}\right)
$$

with the plus (minus) sign describing amplitude (phase) objects. The function $T$ is given by

$$
T\left(\mathbf{q}, q_{z}\right)=\frac{C(\mathbf{q})}{\sqrt{2 \pi}} \frac{\exp \left[-\frac{1}{2}\left(\frac{q_{z}-\bar{q}_{z}(\mathbf{q})}{\Pi(q)}\right)^{2}\right]}{\Pi(q)}
$$

where $\bar{q}_{z}(\mathbf{q})=\frac{q^{2}}{2 k_{0}}\left[1-2\left(\frac{\sigma_{c}}{\sigma_{o}}\right)^{2}-\frac{1}{\sigma_{o}^{2}}\left(\frac{q \Delta \lambda}{2 \pi}\right)\right]$ and $\Pi^{2}(\mathbf{q})=q^{2}\left[\sigma_{c}^{2}+\frac{1}{4}\left(\frac{q \Delta \lambda}{2 \pi}\right)\right]$. The parameters $\sigma_{c}$ and $\sigma_{o}$ account for the numerical apertures of the condenser and the objective, respectively, and their actual relation with the nominal numerical aperture values given by the microscope producer can be determined in various ways. For instance, for the data in Fig. 8, calibration with a known sample provided $\sigma_{o}=0.5 N A_{o b j}$, where the nominal numerical aperture of the objective was $N A_{o b j}=0.85$. As an alternative it is possible to compare the Gaussian modulation transfer 
function used in Ref. [34] with the more realistic modulation transfer function used by microscope producers, which corresponds to an optical system with a uniformly illuminated circular aperture. A numerical comparison of the two expressions provides $\sigma_{o}=0.54 N A_{o b j}$, in good agreement with the estimate obtained by calibration. Similar considerations hold for the numerical aperture of the condenser $\sigma_{c}$. The other parameters of interest in Eq. 29 are the average wavelength $\lambda_{0}$, the average incident wave-vector $k_{0}=2 \pi / \lambda_{0}$, the wavelength spread of the source $\Delta \lambda$, and a time-independent amplitude $C$ [34].

Phase contrast microscopy In phase contrast microscopy the transmitted beam is attenuated by a factor $\beta$ and a phase retardation of $\pi / 2$ is introduced i.e. $E_{t}=j \beta E_{0}$. This is usually achieved in the bench-top microscope by placing a mask in the back focus of the objective lens (see Fig. 4). In a commercial microscope the same result is obtained by placing in the back focal plane of the objective an annular mask, conjugated with an annular aperture placed in the front focal plane of the condenser lens (see Fig. 5). In this case, the image intensity turns out to be proportional to the phase modulation $\sigma_{P}$ introduced by the sample: $i \propto I_{0}\left(\beta+2 \sigma_{P}\right)$. An explicit expression for the PSF of a phase contrast microscope can be derived with approaches similar to the one presented in Ref. [46].

2.4.2. Fluorescence-based microscopy In fluorescence experiments the density $c$ can be identified with the concentration of fluorophores leading to the fluorescence signal. Since emission from different points of the sample is an uncorrelated process and no phase relationship holds between the emitted waves, the contributions from distinct points to the image intensity distribution sum up on an incoherent (intensity) basis. Generally speaking, this simple additivity rule ensures that the condition expressed by Eq. 16 is fulfilled. We note that $K(\mathbf{x}, z)$ can be identified in this case with the standard definition of the 3D incoherent optical point spread function [40].

Wide-field microscopy In a typical wide-field fluorescence microscope the sample is illuminated by an excitation beam according to a scheme similar to that shown in Fig. 5. At a variance with bright-field techniques, a suitable dichroic filter is placed in the collection arm in order to get rid of the excitation beam and to collect only the fluorescent emission from the sample. The PSF of a wide-field microscope is often described by a Gaussian-Lorentzian model [34]

$$
K(\mathbf{x}, z)=\frac{\exp \left(-\frac{2|\mathbf{x}|^{2}}{w_{0}\left(1+\left(z / z_{0}\right)^{2}\right)}\right)}{1+\left(z / z_{0}\right)^{2}}
$$

where $n$ is the refractive index, $\lambda$ an average wavelength and, in analogy with a Gaussian beam, $z_{0}=\pi n w_{0}^{2} / \lambda$ is the Rayleigh range and $w_{0}$ the beam waist. As observed in Ref. [34], as far as the Stokes shift (i.e. the difference between excitation and emission wavelength) is neglected, the PSF of a wide-field fluorescence microscope can be equally obtained as the fully incoherent limit of the bright field microscope PSF.

Confocal fluorescence microscope In a wide-field microscope the overall intensity of the excitation beam is substantially constant along the optical axis. For thick samples this leads to a strong diffused background due to the fluorescent emission from out-of-focus planes. By contrast, in a confocal microscope a stronger rejection of the out-of-plane contributions is achieved by confocal imaging [6]. Quite independently of the specific solution used to obtain confocality (e.g. laser 
scanning or Nipkow disk [6]), the confocal PSF is satisfactorily approximated with a GaussianLorentzian model, valid for a point-like pinhole of negligible size under the paraxial approximation [41]:

$$
K(\mathbf{x}, z)=\left[\frac{\exp \left(-\frac{2|\mathbf{x}|^{2}}{w_{0}\left(1+\left(z / z_{0}\right)^{2}\right)}\right)}{1+\left(z / z_{0}\right)^{2}}\right]^{2}
$$

where $z_{0}, w_{0}, n$ and $\lambda$ are defined as in the previous paragraph. The optical sectioning capability of the confocal microscope is due to the rapid decay of $K(x, z)$ as a function of $z$. In particular, the total intensity associated to a point-like fluorescing particle decreases with the distance $z$ of the particle from the focal plane as $\int d^{2} \mathbf{x} K(\mathbf{x}, z) \sim\left(z / z_{0}\right)^{-2}$. The existence of a well defined optical section strongly influences the image intermediate scattering function which, for small wave-vectors is typically dominated by the fluctuation along the axial direction [41, 33]. An explicit expression for the image intermediate scattering function in a confocal microscope for a system of Brownian particles can be found in Ref. [33].

TIRF microscopy Similar arguments hold also for total internal reflection fluorescence (TIRF) microscopy. In this case only a thin region of the sample is illuminated by the evanescent wave produced by an excitation beam impinging on the interface between sample and the confining slide under total internal reflection conditions [47]. The PSF of a TIRF microscope is often assumed to take the factorised form:

$$
K(\mathbf{x}, z)=K_{2 D}(x) K_{z}(z)
$$

where $K_{2 D}(\mathbf{x})$ is a 2D PSF (typically modelled as a Gaussian function) and where

$$
K_{z}(z)=\exp \left(-z / d_{p}\right)
$$

roughly corresponds to the evanescent wave intensity profile. Here $d_{p}=\lambda /\left(4 \pi \sqrt{n_{1}^{2} \sin ^{2} \theta_{1}-n_{2}^{2}}\right)$ is the corresponding penetration length, $\lambda$ is the excitation wavelength, $\theta_{1}$ is the angle of incidence of the excitation beam and $n_{1}$ and $n_{2}$ are the refractive indices of the substrate and of the sample, respectively [48].

2.4.3. Non-LSI systems The basic idea underlying DFM is very simple: as far as the dynamics is concerned, since the Fourier domain correlation properties of the images are identical to the corresponding correlation properties of the sample (see Eq. 22), one can substantially forget about any details of the imaging process and work on the images "as if they were the sample". Indeed, as pointed out in the previous paragraph, this is true only if the imaging process is such to preserve linearity and space invariance between the sample density and image intensity. In the following a few examples of common microscopy configurations where this property no longer holds are presented.

Dark-field microscopy A prototypical example where the linearity between sample and image fluctuations can be lost is dark-field microscopy. In a dark-field configuration, the illumination beam transmitted by sample is blocked by a spatial filter in the collection optics in such a way that only the light scattered from the sample is collected and can contribute to the image intensity. This corresponds to a homodyne detection scheme, with the intensity on the image plane being proportional to the square amplitude of the scattered field $i \propto|E|^{2}$. Conceptually, the simplest 
implementation of a dark-field microscope is the one reported in Figure 4 with mask c). The collimated illumination beam transmitted by the sample is focused by the objective lens onto an opaque disk located in the objective back focal point [15]. In a commercial microscope, one of the simplest dark-field configurations (typically used in combination with a low-power objective) can be obtained as described in Figure 5 with the pair of masks c). The sample is illuminated with a hollow light cone whose internal angular aperture is larger than the acceptance angle of the objective. In this condition the transmitted illumination beam is not collected by the objective lens and the only contribution to the image intensity comes from the light scattered by the sample.

Under such circumstances, if a system of scattering particles is considered, the electric field on the image plane is obtained as the superposition of fields $E_{n}$ scattered by the individual particles. This leads to an intensity distribution $i \propto \sum_{n} I_{n}+\sum_{n_{1} \neq n_{2}} E_{n_{1}} E_{n_{2}}^{*}$, where $I_{n}$ represents the intensity scattering pattern of the $n$-th particle. It is clear that, while the first term on the left hand side preserves a linear space invariant relation with the particles distribution, the second term introduces in the image a second order contribution. Simple considerations suggest that the last term, which accounts for the interference between the waves scattered by two different particles, becomes negligible if the typical inter-particle distance is larger than the transverse field correlation length $\xi_{T} \sim \lambda / N A_{c}$ (and of the axial correlation length $\xi_{A} \sim \lambda / N A_{c}^{2}$ ), where $N A_{c}$ the is numerical aperture of illumination. In this case there is no fixed phase relationship between the two scattered waves and terms like $E_{n_{1}} E_{n_{2}}^{*}$ with $n_{1} \neq n_{2}$ vanish. Our conclusion is that, if the optical coherence of the illuminating light is low enough, LSI holds in this case too and dark-field microscopy can be used for quantitative DFM of systems of particles that are not too dense. To the best of or knowledge, this has not yet been verified experimentally.

Homodyne polarised light microscopy Similar considerations hold also for the case of polarised light microscopy, when the fluctuations of a birefringent sample (like a nematic liquid crystal or a dense suspension of anisotropic particles) are observed between crossed polarisers [8]. Even in this case, if the average optic axis of the sample is aligned along or perpendicularly to the axes of the polarising elements, there is no transmitted beam and the optical signal is quadratic in the local deformation of the alignment. We note that by careful choice of the orientation of the polarising elements it is still possible to recover the heterodyne regime also in this case [49, 50], as it will be discussed in more detail in Section 3.

\subsection{The structure of a DFM experiment}

The core of a typical DFM experiment consists in the acquisition of a stack of images, usually with a fixed frame rate $\gamma_{0}=1 / \Delta t_{0}$. Fourier domain correlations between the images are then computed and averaged, in order to obtain an estimate of (for instance) the image intermediate scattering function $F_{i}(\mathbf{q}, \Delta t)$. The relevant dynamic parameters of the sample are then obtained by fitting $F_{i}(\mathbf{q}, \Delta t)$ with a suitable model function.

Setting up the microscope and the camera The overall $q$-range where a meaningful information can be obtained from a DFM experiment is determined by variety of factors. In principle, the lowest $q$-value that can be investigated is dictated by the image size as $q_{0}=2 \pi M /\left(N_{0} d_{p i x}\right)$, where $d_{p i x}$ is the pixel size, $M$ is the magnification and $N_{0}$ is the number of rows (or columns) in the image, supposed square. In practice, at the smallest $q$ the system is often very slow and within the experimental duration it is sometimes not possible to observe a complete decorrelation, which 
prevents a statistically accurate determination of the dynamics. Moreover, in many cases of interest (such as bright-field microscopy of phase objects [34]), the low- $q$ signal is strongly depressed by the convergence to zero of the transfer function for $q \rightarrow 0$ and distinguishing the signal from the camera noise can become statistically demanding.

At large $q$ the theoretical upper limit $q_{\max }=\pi M / d_{\text {pix }}$ holds, according to the Nyquist criterion. In practice, a most stringent bound can be imposed by i) the sampling interval $\Delta t_{0}$ between the images, which determines the fastest correlation time $\tau(q)$ that can be reliably measured, ii) the large- $q$ cut-off typically introduced in the transfer function by the finite numerical aperture of the collection optics and the limited coherence of illumination iii) the large- $q$ cut-off possibly introduced by the form factor of the particles [41], in analogy with far-field scattering.

We note that the different relevant experimental parameters that determine the accessible $q$ range are far from being independent. For example, a high frame rate, which is needed to capture high- $q$ fast dynamics, typically requires short exposure times, which in turn lead to a low number of collected photons and thus to a degraded signal to noise ratio, which is particularly tedious close to the upper limit of accessible $q$-range, where the signal vanishes due to the above-cited effects. On the other hand, trying to compensate this effect by increasing the amount of light impinging on the sample via an increase of the condenser aperture, would also accelerate the high- $q$ decay of the transfer function and, in general, it is not obviously beneficial.

Generally speaking, the optimal settings (in terms of objective magnification, coherence of illumination, image size, frame rate, exposure time, duration of the acquisition, ...) for a given DFM experiment are the result of an accurate tradeoff between competing needs.

There are a number of strategies that can be adopted in order to improve the quality of DFM data, in particular in view of overcoming practical instrumental limitations (in the maximum frame rate of the camera, in the maximum number of images that can be acquired/saved, ...). For example acquiring different stacks under the same conditions but with different frame rates, adopting acquisition schemes with variable time delay between the images (multi-tau schemes), or adopting a variable exposure time approach [51] can be all beneficial.

Data processing Once an image stack has been saved, a series of steps brings to the final result i.e. a quantification of the correlation properties of the sample via the computation of the Fourier domain correlation between the images. Once again (see also Sect. 2.3) we stress that the information about the sample that can be extracted from the analysis of the correlation of the image intensity in the Fourier space is substantially independent on the specific representation (intermediate scattering function, structure function, dynamic structure factor, ...) or algorithm (differential/non-differential, time-domain based/frequency-domain based) used for the analysis. In practice, the choice of a specific algorithm or representation is dictated by a variety of factors among which we cite the possibility to establish a direct comparison with an available theoretical prediction, the computational speed/efficiency, the presence of specific forms of noise (periodic/nonstationary), the amplitude of the signal. For instance, a differential approach $[52,27,29]$ and the related structure function are proven to be particularly robust in presence of high mean photon counts [53] or of a systematic time-dependent additive disturbance [54]. The differential approach is also suitable in all those cases for which the individual images contain a strongly non uniform static background contribution $i_{0}(x)$ that introduces in Eq.17 a $q$-dependent term. By contrast, if one is interested in estimating also the background contribution $i_{0}(x)$ it might be more convenient to use a non differential algorithm.

For the analysis of DFM data, Fourier transforming each image is needed. This is conveniently 


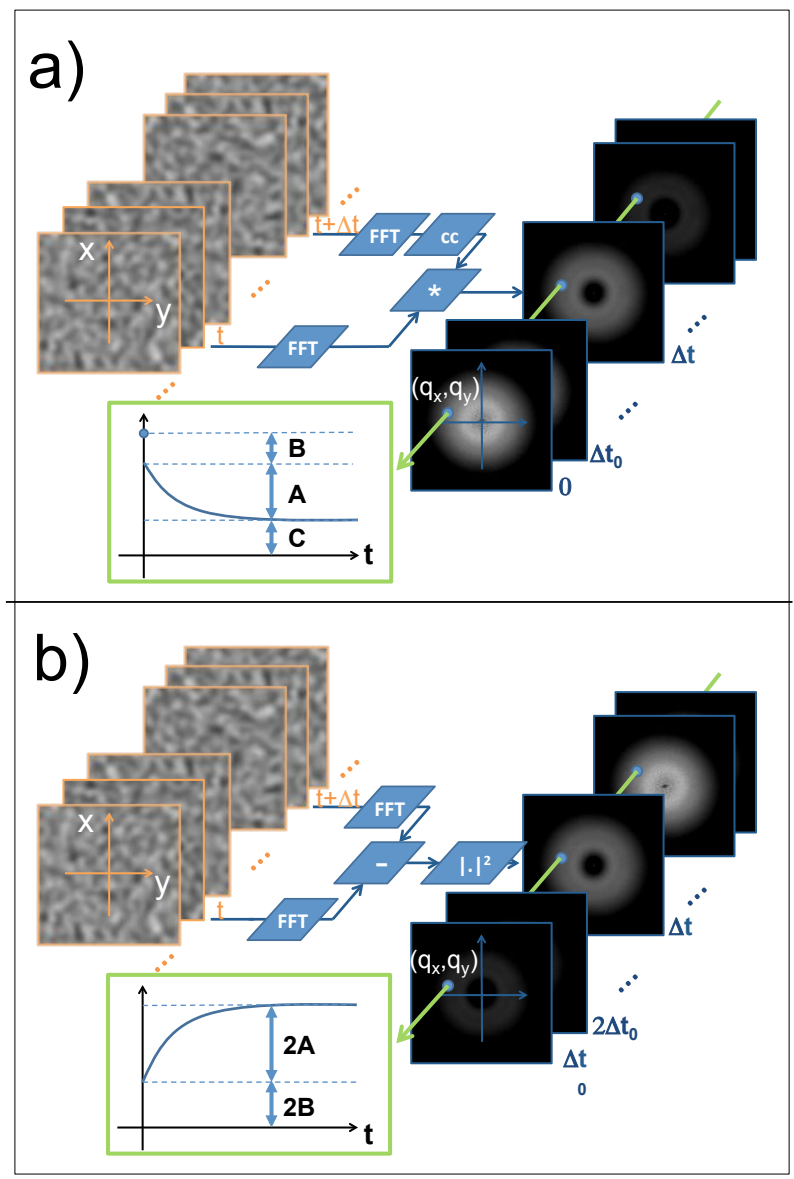

Figure 6. Typical data processing schemes for DFM experiments in time domain. A sequence of $N_{t}$ images is acquired with a fixed frame rate $\gamma_{0}=1 / \Delta t_{0}$. (a) For each $\Delta t$ the image intermediate scattering function $F(\mathbf{q}, \Delta t)$ is calculated as follows. Two images separated by $\Delta t$ are considered and their Fourier cross-spectrum is calculated. This operation is repeated for all the pairs separated by the same $\Delta t$ and the average cross-spectrum provides an estimate for $F(\mathbf{q}, \Delta t)$. For a fixed $\mathbf{q}=\left(q_{x}, q_{y}\right), F(\mathbf{q}, \Delta t)$ is in general a decreasing function of $\Delta t$ described by Eq. 36. (b) For each $\Delta t$ the image structure function $D(\mathbf{q}, \Delta t)$ is calculated as follows. Two images separated by $\Delta t$ are considered and their Fourier transforms are subtracted. The squared amplitude of the result is then calculated. This operation is repeated for all the pairs separated by the same $\Delta t$ and the average value provides an estimate for $D(\mathbf{q}, \Delta t)$. For a fixed $\mathbf{q}=\left(q_{x}, q_{y}\right)$, $D(\mathbf{q}, \Delta t)$ is in general an increasing function of $\Delta t$ described by Eq. 37. 
done by Fast Fourier Transform (FFT) of the pixel matrix $i_{n_{1} n_{2}}$ :

$$
\hat{i}(\mathbf{q}, t)=\left(\frac{d_{p i x}}{M}\right)^{2} \sum_{n_{1} n_{2}} e^{-j \frac{2 \pi}{N_{0}}\left(m_{1} n_{1}+m_{2} n_{2}\right)} i_{n_{1} n_{2}}
$$

where $\mathbf{q}=\frac{2 \pi}{N_{0} d_{p i x}}\left[m_{1} m_{2}\right]$, with $m_{1}, m_{2}$ integers. The following steps depend on the choice of the specific representation of the image correlation. For instance, if the image intermediate scattering function representation is chosen, its best estimate $\bar{F}_{i}(\mathbf{q}, \Delta t)\left(\Delta t=m \Delta t_{0}\right.$, with $m$ integer), can be obtained as an average over all pairs of images separated by $\Delta t$ :

$$
\bar{F}_{i}(\mathbf{q}, \Delta t)=\frac{1}{N_{t}-m} \sum_{n=1}^{N_{t}-m} \hat{i}\left(\mathbf{q},(n+m) \Delta t_{0}\right) \hat{i}^{*}\left(\mathbf{q}, n \Delta t_{0}\right)
$$

where $N_{t}$ is the total number of images of the stack. The operation described in Eq. 35 is typically performed as a matrix operation, i.e. by calculating the same time average simultaneously for all the values of $\mathbf{q}$ (Figure 6a). We note that this is often a highly redundant operation, which is known as greedy sampling [55]. Indeed, since each Fourier component of the image decorrelates with a $q$-dependent characteristic time $\tau(\mathbf{q})$, the actual number of statistically independent samples in the sum on the right hand side of Eq. 35 is in general different for different values of $\mathbf{q}$. This implies that all the $\mathrm{q}$ whose characteristic time exceeds the $\Delta t$ of interest do not benefit from averaging over all the possible pairs separated by $\Delta t$ and the same accuracy could be attained by averaging over a significantly smaller number of images. As discussed in Section 4, the optimisation of this computational step is crucial when a fast analysis is needed [56, 41].

The last step consists in the comparison between the obtained image intermediate scattering function and a model for both the generalised PSF and the sample intermediate scattering function $F(\mathbf{Q}, \Delta t)=S(\mathbf{Q}) f(\mathbf{Q}, \Delta t)$. If the optical system is such that Eq. 22 holds for the q-range of interest, we obtain

$$
F_{i}(\mathbf{q}, \Delta t)=A(\mathbf{q}) f(\mathbf{q}, \Delta t)+B(\mathbf{q}) \delta_{\Delta t, 0}+C(\mathbf{q})
$$

where $\delta_{\Delta t, 0}$ is the Kronecker delta, $A(q)=N|\hat{K}(q)|^{2} S(q), B(q)$ accounts for the camera noise and $C(q)=\left|\hat{i}_{0}(q)\right|^{2}$.

Similar procedures can be followed for other representations of the image correlation such as the image structure function $D_{i}(\mathbf{q}, \Delta t)$ or the dynamic structure factor $S_{i}(\mathbf{q}, \omega)$. For instance, an expression equivalent to Eq. 36 for the image structure function is given by

$$
D_{i}(\mathbf{q}, \Delta t)=2 A(\mathbf{q})[1-f(\mathbf{q}, \Delta t)]+2 B(\mathbf{q}) .
$$

It is worth mentioning that passing from the raw images to the desired correlator in the Fourier space does not necessitate or even necessarily benefit from any additional pre-processing of the raw images, such as for instance background correction, image flattening, smoothing, denoising, stack alignment, etc. By contrast, some of these operations that are useful and have a simple interpretation in the real space might have a disastrous effect in the Fourier space. By contrast, simple pre-processing such image cropping or pixel binning may be helpful in reducing the overall computational speed of the code. It is known that the computational complexity of the 2D FFT is $\mathcal{O}\left(N_{0}^{2} \log N_{0}\right)$, for images made up of $N_{0} \times N_{0}$ pixels, where $N_{0}=2^{j}$ with $j$ integer. If $N_{0}$ can not be obtained as a power-of-two number or is too large, it is thus possible to combine cropping and binning (with obvious consequences on the accessible $q$-range) to obtain a final image that is optimised in pixel size and number. 


\section{Applications}

In the previous section we have tried to present in a more ordered and systematic ways several apparently unrelated methods and approaches that have been introduced during the years by various investigators, interested in a variety of systems. In this section, we will present a focussed selection of the most relevant soft matter systems, whose dynamics has been profitably studied by means of DFM. In doing so, where possible, we will try to be respectful of the historical order of the introduced developments and to describe the distinctive features and range of action of each method.

\subsection{Dynamics in the presence of long-range correlations in molecular and macromolecular systems}

3.1.1. Equilibrium fluctuations Optical inhomogeneities at the molecular level cause measurable light scattering. In the most common case, such inhomogeneities are due to equilibrium fluctuations of the refractive index caused by fluctuations in one or more characteristic variables describing the sample, typical examples being temperature or concentration. The light scattered by a molecular medium is, with a few exceptions, very weak and, despite the progresses in camera technology, it is still practically impossible to perform camera based scattering experiments on molecular systems in equilibrium conditions. A notable exception is represented by molecular scattering close to a critical point or to a phase transition, for which the amplitude of the fluctuations can become so large to be easily seen in microscopy images of the sample. Another molecular system for which fluctuations have a rather large amplitude due to the softness of the system itself is a layer of suitably aligned liquid crystals. The director fluctuations originate intensity fluctuations that can be easily visualised in real space by means of depolarised microscopy and recorded with a camera for subsequent analysis. This idea was originally used in Ref. [57] where the orientational fluctuations in a smectic liquid crystal were successfully observed with a camera. From the series of images obtained, first the spatial Fourier transformation of each image was computed and then the time correlation function at each wave-vector was calculated. From a fitting of the exponentially decaying time correlation function the ratio of the splay elastic constant to the splay viscosity was obtained but the twist and bend ratios could not be estimated. A similar procedure was followed by the same Authors in Ref. [58] for nematic liquid crystals. The Authors were able to extract a twist viscoelastic ratio in agreement with previous depolarised DLS measurements, but no information about the bend and splay viscoelastic ratios could be retrieved. Only very recently [50] it was demonstrated that the use of DDM with polarising elements permits the full characterisation of all three LC viscoelastic ratios in suitably aligned nematics and the unique space-resolving capacities of the proposed method were exploited also to investigate nematics in the presence of spatial disorder, where traditional light scattering fails. Such findings demonstrate that the DFM approach can provide a space-resolved probe of the local sample properties, which is very promising for the study of other optically anisotropic soft materials.

3.1.2. Non-equilibrium fluctuations Large amplitude fluctuations are also observed in molecular systems kept outside of equilibrium by macroscopic external gradients of temperature or concentration. Under such circumstances, equilibrium velocity fluctuations couple with the imposed gradient and cause non-equilibrium fluctuations in the corresponding variable [59].

In some conditions, when the externally applied temperature or concentration gradient overcomes a fixed threshold, the non-equilibrium fluctuations are amplified instead of relaxing back 
by heat or mass diffusion and well known hydrodynamic instabilities such as Rayleigh-Bénard or Soret-driven convection set in [60]. Immediately below the threshold, non-equilibrium fluctuations are largely amplified and DFM methods based on quantitative shadowgraphy [21, 22] have been used profitably for the characterisation of such fluctuations in a single component fluid [21] and in a mixture [61]. For a single component fluid close to the onset of Rayleigh-Bénard convection the dynamics of the fluctuations was also studied [62]. As of today, no experimental measurement of the pre-transitional fluctuations in soft matter systems has been made available, even though a large signal should be expected in light of recent results with colloidal samples [63].

The presence of an instability threshold places an upper limit to the amplitude of the nonequilibrium fluctuations, which increases with the value of the external gradient. A way to bypass this limitation and obtain a larger signal is to operate under gravitationally stable conditions, which allows to apply arbitrarily large gradients. A gravitationally stable configuration is obtained for instance by heating a single component fluid from above (for temperature fluctuations) or by creating a sharp interface between two different fluids (for concentration fluctuations), with the denser fluid at the bottom. In the latter case, it is well known that a carefully prepared sharp interface between miscible fluids becomes progressively smeared in time because of diffusion. However, it has been shown only recently that this apparently quiet remixing is accompanied by giant concentration fluctuations, whose size on Earth is limited only by gravity [64]. On Earth, the amplitude and the dynamics of such fluctuations have been successfully characterised with traditional scattering [64] but for this kind of systems shadowgraphy has been proven to outperform traditional scattering both for static [65] and dynamic [66] scattering measurements.

For this reason, shadowgraphy was also chosen for performing non-equilibrium experiments in space, where the microgravity conditions provided the ideal environment for obtaining fully developed concentration fluctuations in a macromolecular system [67, 68]. The study of these fluctuations was justified by its potential impact on the understanding of the growth of materials in space, in particular for protein crystals [69] for which it is suspected that the non-equilibrium fluctuations may be responsible of imperfect crystallisation in microgravity [68]. Images of the concentration fluctuations obtained in microgravity, limited only by the size of the container, are shown in Fig. 7 for a dilute polymer solution of polystyrene in toluene subjected to a Soretinduced concentration gradient that increases in time. It is evident that the fluctuations in space are way larger in amplitude than those observed under the same conditions on Earth (see caption for additional details).

The unique visualisation opportunity offered by imaging methods compared with traditional scattering did not come at the expenses of quantitativeness. Indeed, by DFM analysis it was possible to extract the amplitude (only after suitable calibration of the transfer function with known colloidal particles) and the characteristic time of the fluctuations (see Fig. 7), which confirmed theoretical expectations for long-ranged, long-lived non-equilibrium fluctuations enhanced by the absence of gravity [59].

\subsection{Dynamics of colloidal systems}

A dilute colloidal suspension of noninteracting spherical particles represents the ideal benchmark system for testing the capabilities and the performances of a novel scattering technique. This is mainly due to the availability in the market of well characterised, mono-disperse spheres of various materials in different dispersing media but also to the fact that there are well confirmed theoretical predictions for the static and dynamic scattering from such a suspension. So, it is not surprising 



$0 \mathrm{~s}$
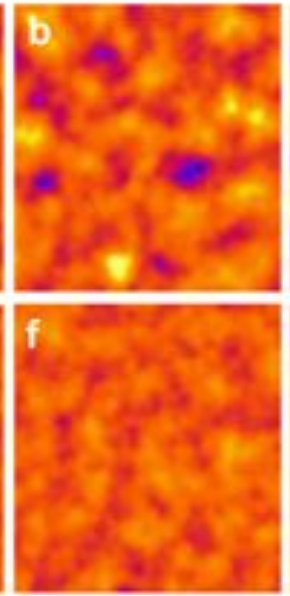

$500 \mathrm{~s}$
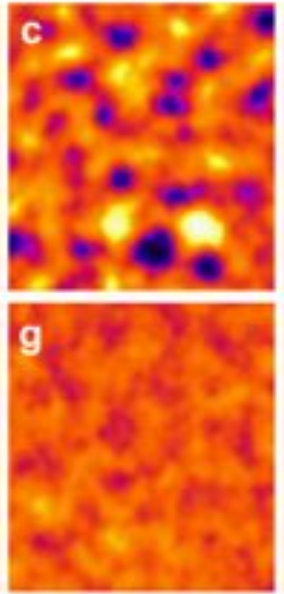

$1000 \mathrm{~s}$

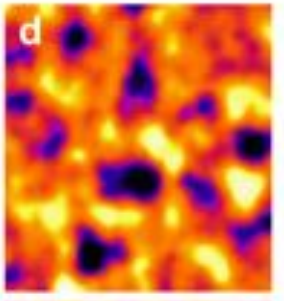

h

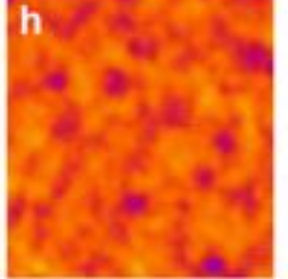

$2000 \mathrm{~s}$
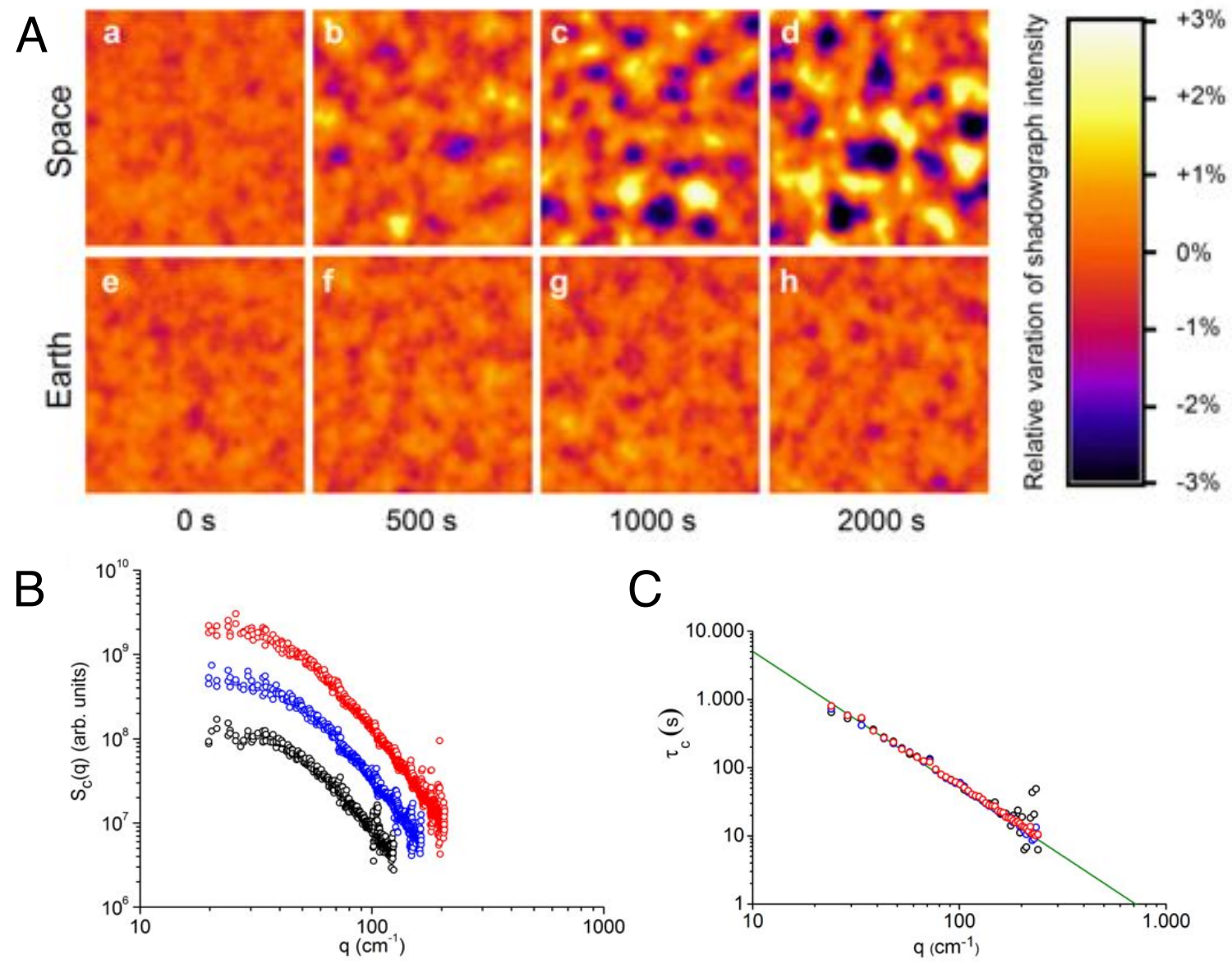

C

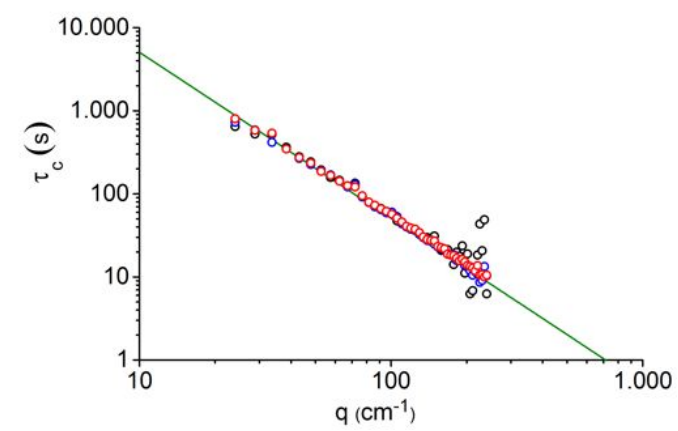

Figure 7. Non-equilibrium fluctuations in a polymer solution of polystyrene in toluene. (A) False-colour shadowgraph images of non-equilibrium fluctuations in microgravity (a-d) and on Earth (e-h). Images were taken $0,400,800$, and 1,600 s (left to right) after the imposition of a $17.40 \mathrm{~K}$ temperature difference that creates non-equilibrium concentration fluctuations due to Soret effect. The side of each image corresponds to $5 \mathrm{~mm}$. Colours map the deviation of the intensity of shadowgraph images with respect to the time-averaged intensity. (B) Meansquared amplitude of non-equilibrium concentration fluctuations in microgravity for three sample subjected to the presence of temperature differences of $4.35 \mathrm{~K}$ (black circles), $8.70 \mathrm{~K}$ (blue circles) and $17.40 \mathrm{~K}$ (red circles). (C) Relaxation time of non-equilibrium concentration fluctuations as a function of wave-vector. The black data correspond to a temperature difference of $4.35 \mathrm{~K}$, the blue data to $8.70 \mathrm{~K}$ and the red ones to $17.40 \mathrm{~K}$. The solid line represents the diffusive time $\tau_{c}=1 /\left(D_{0} q^{2}\right)$ as estimated from literature data for the diffusion coefficient $D_{0}$. Reprinted (adapted) with permission from Ref. [68]. Copyright (2011) Nature Publishing group. 

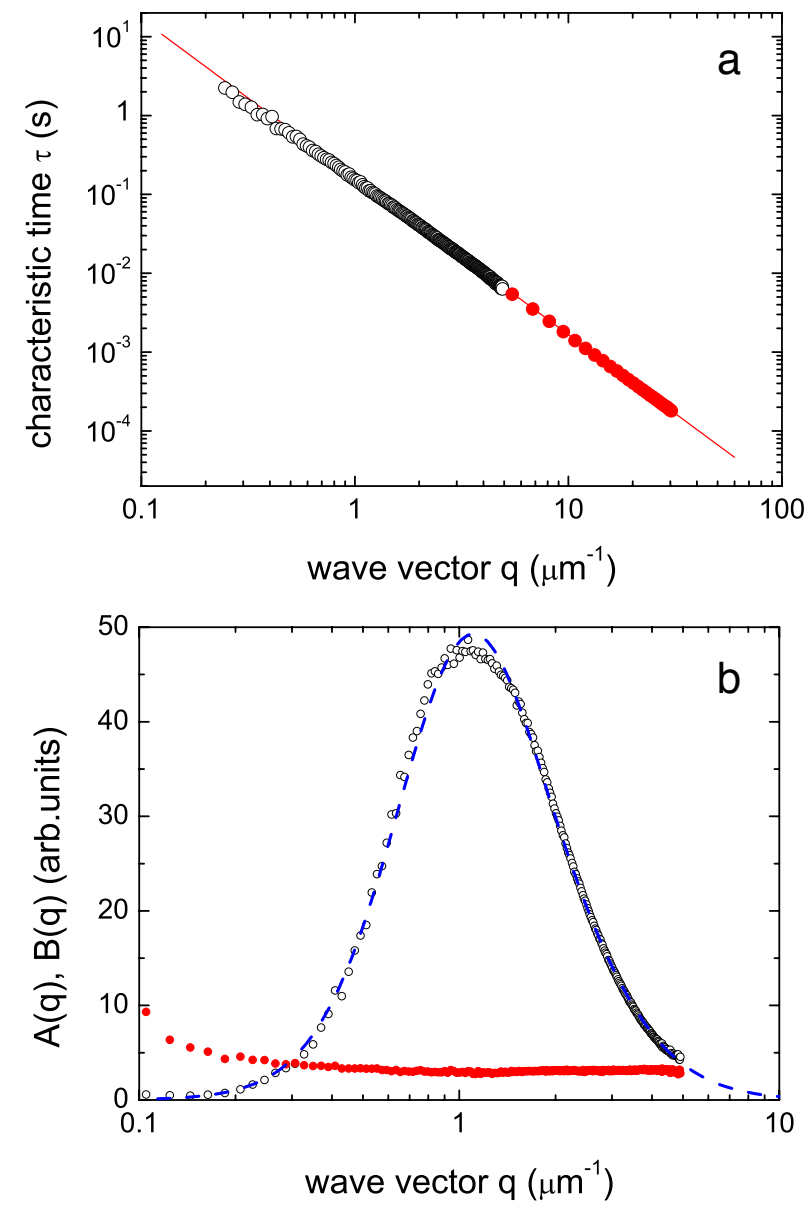

Figure 8. Experimental results of DFM experiments performed in on a dilute colloidal suspension of a $73 \mathrm{~nm}$ (diameter) polystyrene particles in water. (a) Experimentally determined characteristic decay time $\tau$ plotted against the wave-vector q. Open (black) circles are data obtained with brightfield DDM. Close (red) circles are DLS data. The continuous line in the graph is the theoretical estimate corresponding to $D_{0}=6.0 \mu \mathrm{m}^{2} / \mathrm{s}$. (b) Experimentally determined $A(q)$ and $B(q)$ (see Eq.37) plotted against the wave-vector $q$. The dashed line is a fit of the data with a model based on Eq. 29. Reprinted figures with permission from Ref. [34]. Copyright (2011) by the American Physical Society.

that many of the DFM experimental methods - for both scattering-based and fluorescence-based DFM - have been initially validated with a dilute suspensions.

We show in Fig. 8 the results of a bright-field DDM experiment on a colloidal suspension of polystyrene particles with diameter equal to $73 \mathrm{~nm}$, confined in a capillary tube with rectangular section of thickness $100 \mu \mathrm{m}$. Measurements were taken with a commercial microscope set-up, as specified in Ref. [34]. The signal from the particles (empty symbols) is well above the noise (full symbols) (Fig. 8b) and the typical range in which the correlation time could be estimated amounts to about a factor of 20 in $q$ (Fig. 8a), which should not be meant as a general limit of the approach 


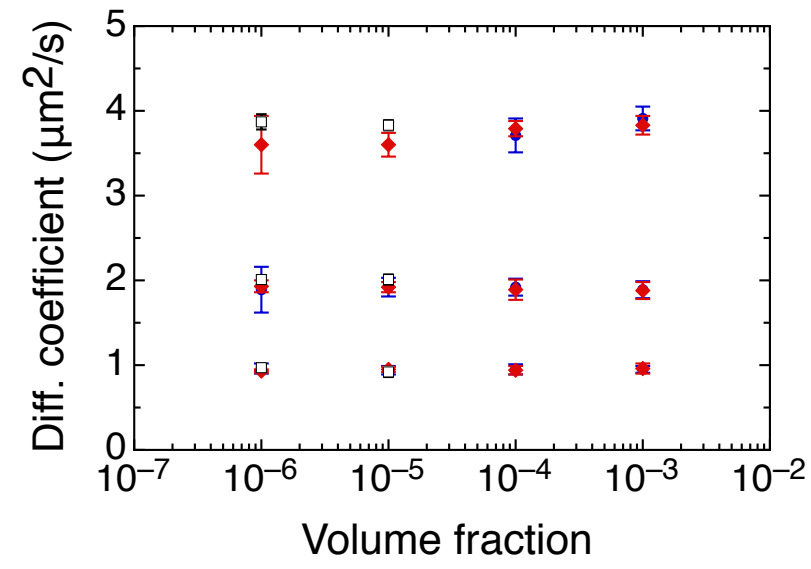

Figure 9. Diffusion coefficients measured in Ref. [70] by using b-DDM (red full diamonds), f-DDM (blue full circles) and DLS (black open squares) for aqueous suspensions of nanoparticles with diameters (from top to bottom) $100 \mathrm{~nm}, 200 \mathrm{~nm}$ and $400 \mathrm{~nm}$

but rather as the outcome of the typical experimental parameters employed here. In the accessible q-range, scattering from the particles is featureless and we can interpret the curve in Fig. 8b as an experimental measurement of the transfer function for this microscope set-up [34].

An interesting comparison between bright-field DDM (b-DDM), fluorescence-based DDM (fDDM) and DLS was performed in Ref. [70]. Colloidal dispersions of particles with diameter 100, 200 and $400 \mathrm{~nm}$ were prepared at different volume fractions $\left(10^{-6}, 10^{-5}, 10^{-4}\right)$ and studied with the three techniques.

The results (Fig. 9) show that, within the experimental errors, all the measurements for the dynamics are in excellent agreement, confirming thereby that DFM approaches based on different imaging processes are substantially equivalent. It should be however noted that the results obtained for the statics in b-DDM and in f-DDM are different, due to the expected differences in the transfer function of the two techniques. While the transfer function for b-DDM presents the same features of the one shown in Fig. 8, the transfer function for f-DDM does not exhibit the drop of signal at small $q$, as expected from theory (see Section 2.3 and Ref.[34]).

One of the interesting possibilities offered by DFM - especially with the structure function approach - is the capability of subtracting unwanted static features from the sample image. This feature was exploited in Ref. [71], where the diffusive dynamics of colloidal particles was studied in a landscape made of micro-fabricated arrays of nano-posts of diameter $500 \mathrm{~nm}$, spaced by 1.2-10 $\mu$ $\mathrm{m}$ on a square lattice.

Measurements were performed by DDM and showed that, as the spacing between posts was decreased, the dynamics of the nanoparticles slowed (Figure 10) and was increasingly better represented by a stretched exponential rather than a simple exponential. Such findings suggest that further increasing the confinement could lead to either dynamic heterogeneity or possibly even vitrification. In the same article it was also shown that the diffusion coefficients extracted from video particle tracking were in excellent agreement with those obtained from the DDM measurement on $400 \mathrm{~nm}$ particles, confirming that the two analyses of the microscopy data probed the same diffusive behaviour.

However, we note that while single particle tracking probes the self diffusion of particles, all 

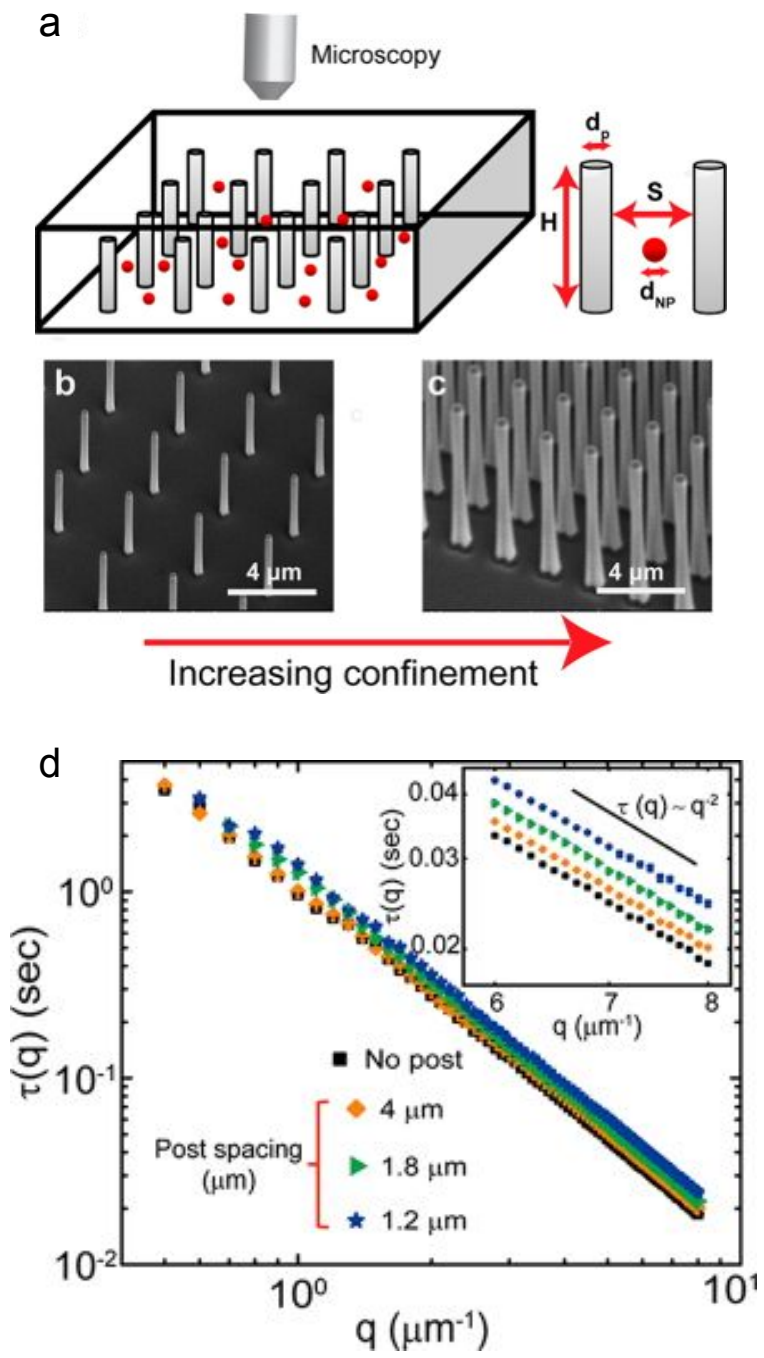

Figure 10. DDM experiments on confined colloidal particles. (a) Cylindrical post arrays filled with a polystyrene nanoparticle suspension. (b, c) Scanning electron micrographs of posts of diameter $d_{p}=500 \mathrm{~nm}$, (b) height $H=10 \mu \mathrm{m}$ and spacing $S=6 \mu \mathrm{m}$, and (c) height $H=11.9$ $\mu \mathrm{m}$ and spacing $S=1.6 \mu \mathrm{m}$. (d) Relaxation time $\tau(q)$ as a function of wave-vector $q$ for 400 $\mathrm{nm}$ nanoparticles diffusing in the bulk (black squares) and in post arrays with $S=4 \mu \mathrm{m}$ (orange diamonds); $S=1.8 \mu \mathrm{m}$ (olive right triangles); and $S=1.2 \mu \mathrm{m}$ (navy stars). The inset shows that $\tau(q)$ scales as $q^{-2}$ over the range of wave-vectors from $q=6$ to $8 \mu m^{-1}$. The error bars for $\tau(q)$ are smaller than the symbols. Reprinted (adapted) with permission from Ref. [71]. Copyright (2013) American Chemical Society. 


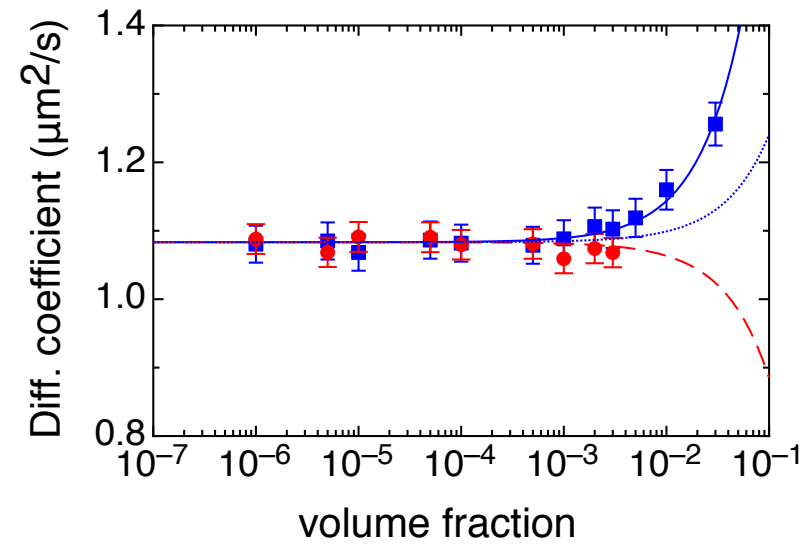

Figure 11. Diffusion coefficients of colloidal suspensions of $440 \mathrm{~nm}$ (diameter) particles as a function of the volume fraction $\phi$ of the particles (unpublished results). For each $\phi$, the same phase-contrast movie is analysed by both DDM and SPT and the short-time diffusion coefficient is extracted. Collective diffusion coefficients $D_{c}(\phi)$ obtained with DDM are plotted as blue squares, whereas self diffusion coefficients $D_{s}(\phi)$ obtained with SPT are reported as red circles. The blue dotted line is the theoretical prediction for the short-time collective diffusion coefficient of hard spheres $D_{c}(\phi)=D_{0}(1+1.45 \phi)$. The red dashed line is the theoretical prediction for the shorttime self diffusion coefficient of hard spheres $D_{s}(\phi)=D_{0}(1-1.831 \phi)$. The continuous blue line is a linear fit of the DDM data that provides the best estimate $D_{c}(\phi)=D_{0}(1+5.6 \phi)$. In all cases the single particle diffusion coefficient was $D_{0}=1.083 \mu \mathrm{m}^{2} / \mathrm{s}$.

DFM methods are mostly sensitive to collective diffusion i.e. to the way a sinusoidal modulation of the density relaxes back by diffusion. Only for very dilute samples the collective diffusion coefficient $D_{c}$ and the self-diffusion coefficient $D_{s}$ become identical with the Stokes-Einstein prediction $D_{0}$ for a single particle. For interacting particles they are expected to be markedly different, as a result of the interactions.

This can be appreciated in Fig. 11, where we report unpublished results of experiments performed at various volume fractions $\phi$ on colloidal suspensions of charged polystyrene particles with nominal diameter $440 \mathrm{~nm}$. The same movies were analysed with both phase-contrast DDM and single-particle tracking (SPT). For dilute samples the two methods give the same result but, with increasing volume fraction, the inter-particle repulsive interactions are expected to speed-up collective diffusion and slow down self-diffusion. However, SPT experiments above $\phi=3 \times 10^{-3}$ were made difficult by the particle crowding and no reliable estimate for $D_{c}$ could be extracted. By contrast, with DDM it was possible to extract $D_{c}$ for samples that were one order of magnitude more concentrated. As it can be appreciated in Fig. 11, SPT does not provide evidence that the sample behaves differently from a suspension of hard-spheres for which it is expected that $D_{s}(\phi)=D_{0}(1-1.831 \phi)$. On the contrary, DDM results rule out this possibility because the data are clearly incompatible with the theoretical prediction $D_{c}(\phi)=D_{0}(1+1.45 \phi)$. A linear fit with $D_{c}(\phi)=D_{0}(1+\lambda \phi)$ provides the estimate $\lambda=5.6 \pm 0.3$ for the interaction parameter, which is greater than 1.45. as expected for repulsive interactions between charged particles [72]. This example shows the high potential of DFM methods in crowded environments, where tracking becomes extremely difficult if not impossible.

Another interesting application of DFM is the study of the dynamics of anisotropic colloids. In this respect we mention the DFM experiments performed in Ref. [49] on optically anisotropic 


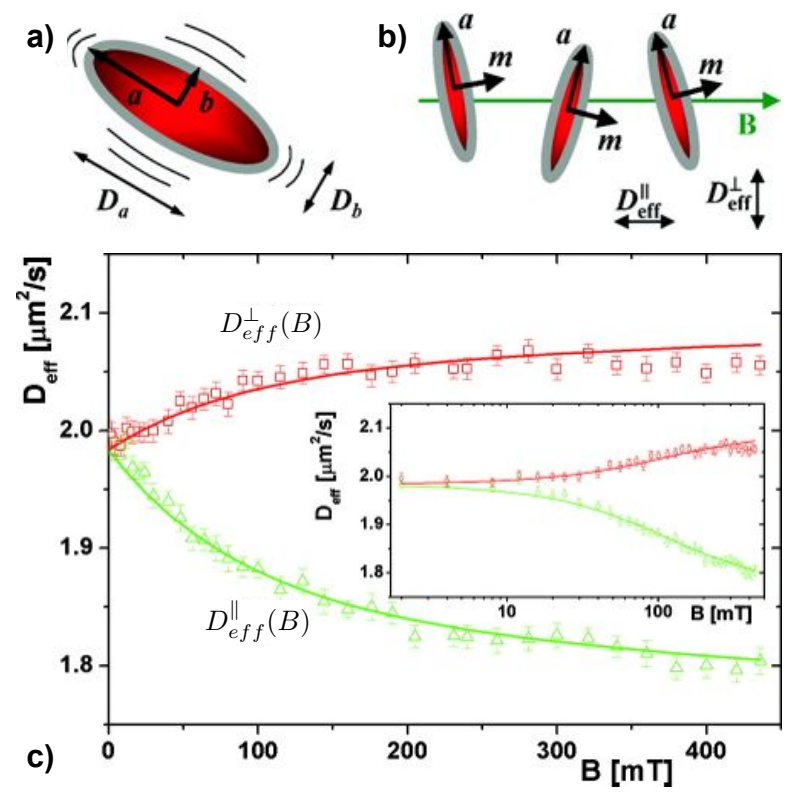

Figure 12. Results of DDM experiments on silica-coated spindle-type hematite particles in the presence of a magnetic field $B$. (a) A single particle with definition of semiaxes and corresponding translational diffusion coefficients. (b) Ensemble of partially aligned particles with definition of effective diffusion coefficients parallel and perpendicular to the applied magnetic field B. (c) Measured effective diffusion along the field direction (green triangles) $D_{e f f}^{\|}(B)$ and perpendicular to the field (red squares) $D_{\text {eff }}^{\perp}(B)$. The lines are the results of fitting the data with a theoretical model described in Ref. [74]. The inset shows the same data in semilogarithmic representation. Reprinted (adapted) with permission from Ref.[74]. Copyright (2012) American Chemical Society.

PTFE and on rod-like iron oxide colloidal particles. By placing the sample between two polarisers whose main axes were reciprocally oriented at an appropriate angle to ensure that the measurements were performed in the heterodyne regime, the translational and rotational diffusion coefficient of the particles were determined. Interestingly, the measurements were performed with a slow camera by varying the exposure time and keeping the delay between the images fixed [51]. This method is the $q$-resolved generalisation of the so called speckle visibility spectroscopy [73].

DFM methods are ideal also for the study of anisotropic colloids under external fields, as shown in Ref. [74]. A dilute aqueous suspension of anisotropic magnetic particles was studied in the presence and in the absence of a magnetic field.

The particles were silica-coated spindle-shaped hematite particles with semiaxes $a=175 \mathrm{~nm}$ and $b=51.9 \mathrm{~nm}$, respectively, which align perpendicular to an applied magnetic field $B$ (Figure 12). Measuring at wave-vectors such that $q a<1$, the Authors ensured that the dynamics was dominated by translational rather than rotational diffusion. Without applied magnetic field, the particles were randomly orientated with isotropic dynamics, whereas with the field the measured translational diffusion was found to decrease in the direction parallel to the magnetic field and increase perpendicular to it (Figure 12). From a measurement of the diffusion along these two directions as a function of magnetic field the Authors extracted the orientational order parameter 
of the system, a quantity normally extracted from static measurements. We will see in he next paragraph another application of this method to magnetic field sensitive bacteria.

A last application of DFM methods that is worth mentioning for its relevance in basic and applied science is the characterisation of colloidal aggregation. DDM has been used profitably to follow the kinetics of an aggregation process induced by destabilising a colloidal suspension of $73 \mathrm{~nm}$ (diameter) particles [75]. Despite the sub-diffraction size of the colloidal particles, the process could be monitored and the hydrodynamic radius of the aggregates was obtained in a time-resolved fashion. The power law growth of the gyration radius as a function of time allowed extracting a fractal dimension of 1.85, compatible with a diffusion limited aggregation process. Similar studies were performed with HNFS in space for aggregation of colloids induced by critical Casimir forces [76]. In that case also the gyration radius could be extracted owing to a calibration of the instrumental transfer function, following the procedure in Ref. [68]. The good results obtained proved that HNFS was an ideal tool for static and dynamic measurements of aggregation of nanoparticles in space.

\subsection{Motility of microorganisms}

DFM has been repeatedly used for characterising the motility of a population of microorganisms. Compared to tracking, it delivers motility parameters with high throughput in a few minutes. With respect to DLS, it offers access more readily to the larger length scales for the microorganisms' motility. As of today, several experiments have been performed with bacterial suspensions (Escherichia Coli [77, 78, 79, 80], Bacillus Subtilis [41] and Magnetospirillium gryphiswaldense [81]) and with a suspension of the alga Chlamydomonas reinhardtii [78]. In all these cases, accessing large length scales is fundamental for capturing the dynamic properties of the microorganisms and is extremely difficult with traditional DLS.

Peritrichously flagellated bacteria such as E. Coli and B. Subtilis, swim by alternating smooth runs (with flagellar motors spinning counterclockwise) with reorienting tumbles (with clockwise spinning). A ballistic run phase takes place approximately at constant speed $v_{0}$ and tumbling events occur with a fixed rate and cause a full randomisation of the direction of motion of the bacteria. In this case, the simplest model for the intermediate scattering function $F(\mathbf{Q}, \Delta t)$ at short times and small $Q$ is that of a 3D ballistic swimming, characterised by $F(\mathbf{Q}, \Delta t)=\sin \left(Q V_{0} \Delta t\right) / Q V_{0} \Delta t$ (see Table 1, column three). For long times, the behaviour becomes diffusive with an effective diffusion coefficient $D$ and $F(\mathbf{Q}, \Delta t)=\exp \left(-D Q^{2} \Delta t\right)$ (see Table 1, column one). Experiments with DFM provide unique access to the small $q$ regime, indispensable for probing unambiguously the bacterial swimming [77]. Experiments on dilute bacterial suspensions [77, 78] assumed a non-tumbling model with Schulz distribution for the bacteria velocity and extracted the average swimming speed $\bar{v}$, the variance $\sigma^{2}$ of the distribution, the motile cell fraction $\alpha$ and the diffusion coefficient $D$. An example of results obtained with wild-type bacteria is reported in Figure 13.

It can be noticed that the average swimming speed in Fig. 13 exhibits a slight decrease towards low $q$, which according to later molecular dynamics simulations [82] seems to be attributable to the effect of tumbling, neglected in the analysis of the experimental data.

Experiments on more concentrated suspensions were made possible by the use of confocal microscopy [41] and showed that, for dense bacteria in the bulk, the data (Figure 14) were well fitted to a compressed exponential $F_{i}(\mathbf{q}, \Delta t)=\exp \left[-\left(q v_{0} \Delta t\right)^{1.35}\right]$, similar to ageing gels and glasses [83]. By contrast, bacteria swimming near the coverslip did not exhibit a well-defined velocity distribution. It would be interesting to compare the experimental results in Ref. [41] with 




Figure 13. Results of DDM experiments on a dilute suspension of wild-type Escherichia Coli. The extracted parameters of the bacterial motion are (from top to bottom): the average swimming speed $\bar{V}$ and the standard deviation $\sigma$ of the Schulz distribution of swimming speeds, the motile fraction $\alpha$ and the diffusion coefficient $D$. Reprinted figure with permission from Ref. [77]. Copyright (2011) by the American Physical Society.

recent theoretical results in Ref. [84], where a very useful analytic expression for $F(\mathbf{Q}, \Delta t)$ was provided and numerical results are obtained for the motion of bacteria close to surfaces.

Finally, DDM was also used to measure the swimming speed distribution and the oscillatory dynamics for C. reinhardtii [78] and for determining, by using the same analysis performed for anisotropic colloids under magnetic field discussed above, the magnetic moment of the magnetic field sensitive Magnetospirillium gryphiswaldense [81].

\subsection{Cell motility}

As far as cells are concerned, the most impressive DFM experiment is the one described in Ref. [85]. The Authors studied the dynamics of monolayers of epithelial Madin-Darby canine kidney (MDCK) cells on a substrate by characterising the cell motion over hours during the growth of the confluent cells and the corresponding increase of the cell density. As the density increased, cellular displacement was progressively inhibited, without any particular hint of structural spatial ordering. However, dynamic heterogeneity was increasingly more evident as time passed, with a sort of microphase separation between fast and slow cells. The intriguing analogy to dynamic heterogeneities found in colloidal systems as they approach a glass transition was particularly evident in the dynamic structure factors of the confluent cell layer (Fig. 15) that exhibited soft modes typical of jammed packing.

To describe the data, the Authors used the damped harmonic oscillator (DHO) model, in which the dynamic structure factor $S(q . \omega)$ is written as the sum of a Rayleigh contribution, which quantifies diffusion, and a Brillouin one that mirrors the elastic response to density fluctuations 


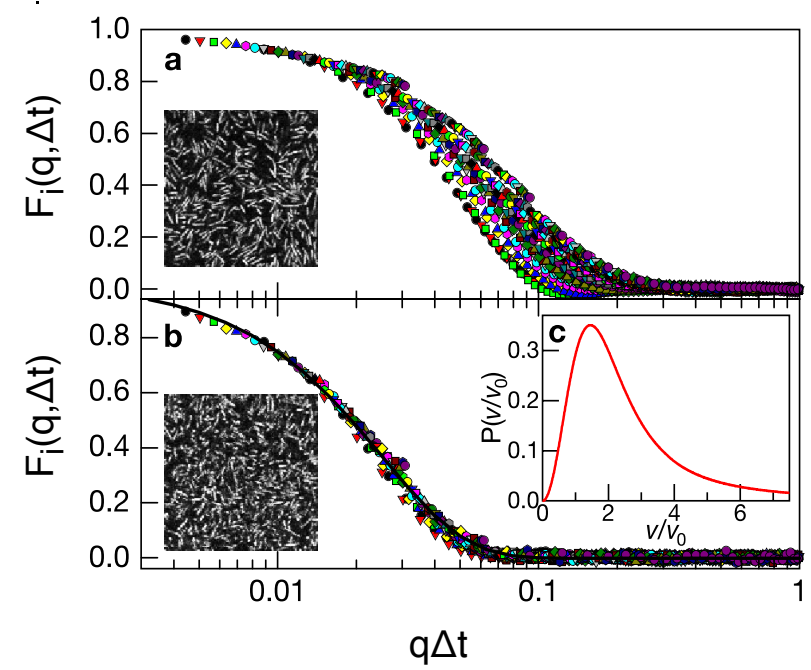

Figure 14. Experimental results obtained with confocal DDM of dense bacteria (Bacillus subtilis) (a) $F_{i}(q, \Delta t)$ and image (inset) for bacteria swimming at the coverslip, for 43 values of $q$ in the range $0.2<q<4 \mu m^{-1}$, each plotted with different symbols, as a function of rescaled time delay $q \Delta t$. (b) $F_{i}(q, \Delta t)$ for bacteria swimming deep in the bulk, $16 \mu \mathrm{m}$ from the coverslip, in the same $q$ range as in (a); here, data from all 43 values of $q$ (symbols) scale onto a single master (solid black) curve of the form $\exp \left[-\left(q v_{0} \Delta t\right)^{1.35}\right]$, with $v_{0}=39.6 \pm 0.3 \mu \mathrm{m} / \mathrm{s}$. (Inset) image of bacteria deep in the bulk, $8 \mu \mathrm{m}$ from the coverslip. (c) Population velocity distribution $P\left(v / v_{0}\right)$ for the bacteria in (b). Reprinted figure with permission from Ref. [41]. Copyright (2012) by the American Physical Society.

(Figure 15B). The width of the central Rayleigh peak is given by $\Gamma_{0}(q)=D_{0} q^{2}$, and the diffusivity $D_{0}$ extracted from the experimental data as a function of the cell occupancy is shown in Figure 15D. Analysis of the Brillouin peaks in $S(q . \omega)$ yields the dispersion relation $\Omega(q)$ of the cell motion at each cell density, from which the density of states is extracted (Figure 15C). Results for $D_{0}$ suggest that cells behave as moderately fragile glasses, whereas the analysis of the density of states identifies two density dependent peaks, reminiscent of boson peaks in supercooled fluids, where they are originated from damped oscillations of long lived molecular structures.

\subsection{Intra-cellular processes}

To our knowledge, the first DFM studies of transport phenomena in cells was reported in Ref. [33], where k-space image correlation spectroscopy (kICS) was used to measure the transport dynamics of a5-integrin/enhanced green fluorescent protein constructs in a transfected $\mathrm{CHO}$ epithelial cell. The measurements, performed in total internal reflection fluorescence (TIRF) microscopy provided a proof that, even in the presence of photobleaching, DFM methods can provide accurate results. Indeed, an effective diffusivity coefficient $D_{\text {eff }}=0.0096 \pm 0.0002 \mu \mathrm{m}^{2} / \mathrm{s}$ and a photobleaching rate $K=0.008 \pm 0.001 s^{-1}$ were independently extracted from the measurements.

Some results on cellular processes were also obtained by the already introduced FTLS. Dynamic FTLS measurements were performed on the fluctuating membranes of red blood cells. The power spectrum was found to follow power laws with different exponents in time for all scattering wavevectors and the cells are found to be more compliant at longer spatial wavelengths [30]. FTLS 

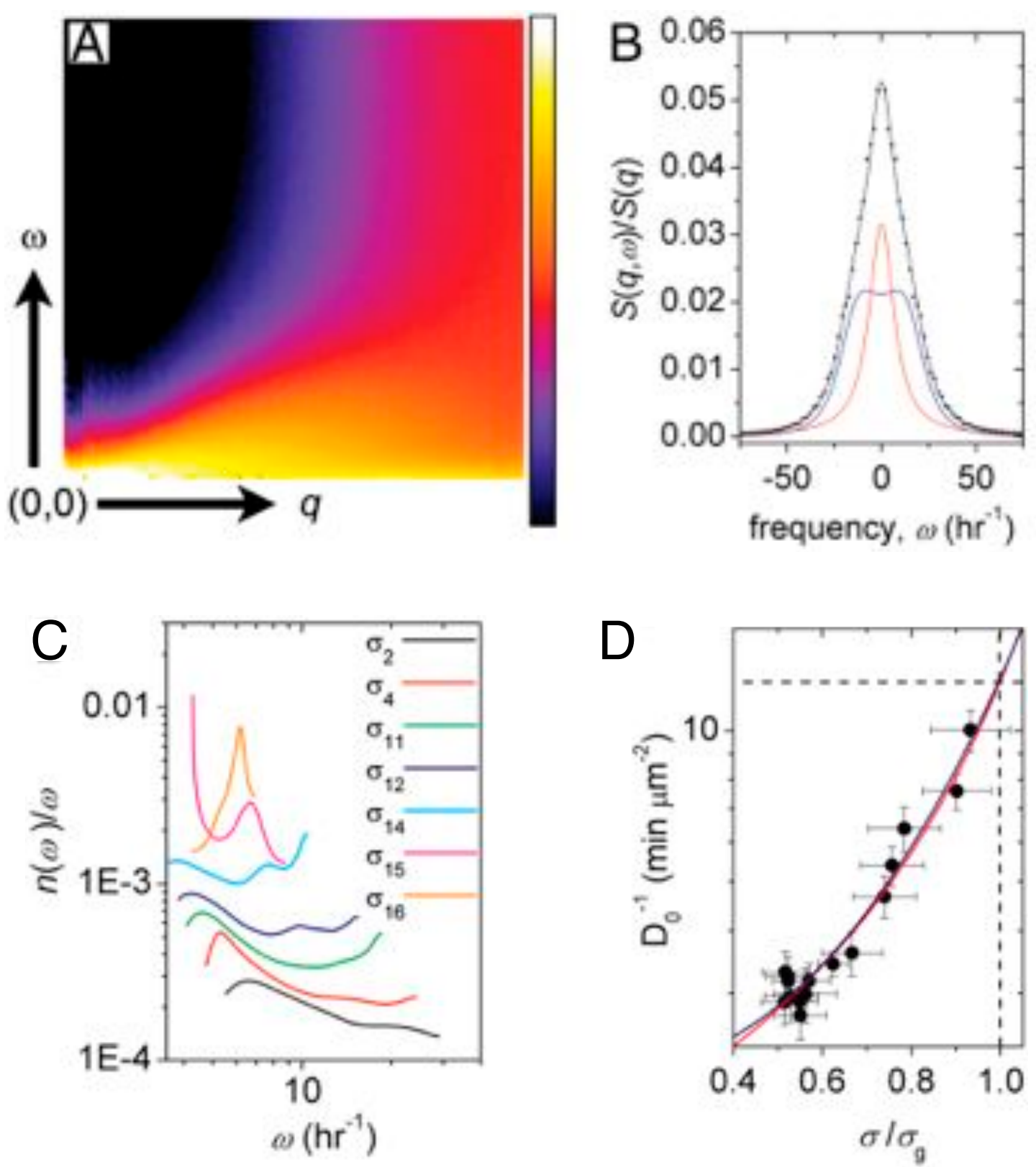

Figure 15. Results of DFM experiments performed on MCDK cells moving on a substrate. (A) The dynamic structure factor $S(q \cdot \omega)$ of the migrating cell monolayer is calculated to quantify cooperative and self motions over a broad range of length scales and time scales. (B) An example slice through $S(q, \omega)$ at $q=0.8 \mu m^{-1}$ shows that the spectral line shape is well described by the DHO model, consisting of a diffusive Rayleigh peak (red line) and a Brillouin peak (blue line). (C) The density of states of cell motion, extracted from the Brillouin scattering, exhibits two sets of peaks, analogous to boson peaks in supercooled fluids. (D) An Arrhenius plot of $D_{0}^{-1}$ (as extracted from the width of the Rayleigh peak) as a function of cell density show a behaviour reminiscent of colloidal glasses. Fitting with theoretical model (lines) suggest that cells behave as moderately fragile glasses. Reprinted figure with permission from Ref. [85]. Copyright (2011) by the National Academy of Sciences. 
was also used to measure the spatio-temporal behaviour of active dynamics due to F-actin in single glial cells. The active contribution of actin cytoskeleton was obtained by modulating its dynamic properties via Cytochalasin-D, a drug that inhibits actin polymerisation/depolymerisation. Even though some differences were observed before and after treatment with Cyto-D, no really quantitative estimators of such differences were provided [86].

Another recently introduced fluorescence-based DFM method is Dispersion-Relation Fluorescence Spectroscopy [87], which is equivalent to the previously mentioned kICS and f-DDM. The authors of Ref. [87] studied mouse embryonic fibroblasts with their actin cytoskeleton labeled with GFP and were able to distinguish between directed and diffusive transport along the actin fibers in living cells. The fluorescently labeled actin cytoskeleton exhibited active transport motion along a direction parallel to the fibers and diffusive in the perpendicular direction, consistently with the phenomenon of "treadmilling" in which actin filaments are continually polymerising at one end and depolymerising at the other.

\subsection{Flow characterisation}

A last example of application of DFM in soft matter is provided by reciprocal space velocimetry [88, 89, 90, 91, 92]. Similar to Particle Imaging Velocimetry [93] and Laser Speckle Velocimetry [94], which however operate in the real space, DFM velocimetry is not based on tracking but on the presence of translating fluctuations of arbitrary origin. Such fluctuations can be due to subdiffraction tracers but also to the sample itself, with no added tracers, provided that it displays sufficiently strong intrinsic refractive index fluctuations [92]. Originally demonstrated in the laserbased HNFS configuration [88, 89, 90], DFM velocimetry operates in the reciprocal space by using the structure function reported in Table 1 for motion with uniform velocity. Equivalently, it can operate in the real space by cross correlation of images at different times. One of the biggest advantage of DFM velocimetry is that it can directly quantify the number of moving particles because it is a heterodyne method. The method was later successfully applied also in b-DDM configuration [91, 92], where the use of a light source with tuneable spatial coherence demonstrated immediate advantages by allowing passing from a $2 \mathrm{D}$ velocimetry to a $3 \mathrm{D}$ one [92].

Indeed, in Ref. [92] the depth resolving capabilities of partially coherent illumination typical of bright field microscopy were used to obtain the velocity profile not only in the transverse direction but also along the optical axis (Figure 16). The obtained axial resolution $\left(\delta z \sim \lambda /\left(N A_{c}\right)^{2} \sim 20\right.$ $\mu m$ ) is still somehow poorer than confocal microscopy but the payoff of the method is that does not require anything more than a commercial microscope.

\section{Conclusion and outlook}

In this review article we have introduced the working principles of Digital Fourier Microscopy, a rather novel set of methodologies that share the interest for the correlation properties of a sample in the reciprocal space. We have provided a set of applications of DFM, with a focus on the dynamics of soft materials. We conclude this review by outlining the areas that in the near future will most likely benefit of DFM methods and of their interesting mix of properties.

Quantitative microrheology A very promising application for DFM is microrheology. Microrheology is a tool for the study of the viscoelastic properties of soft materials, which is based on measuring the dynamics of colloidal particles embedded in a material of interest and extracting 


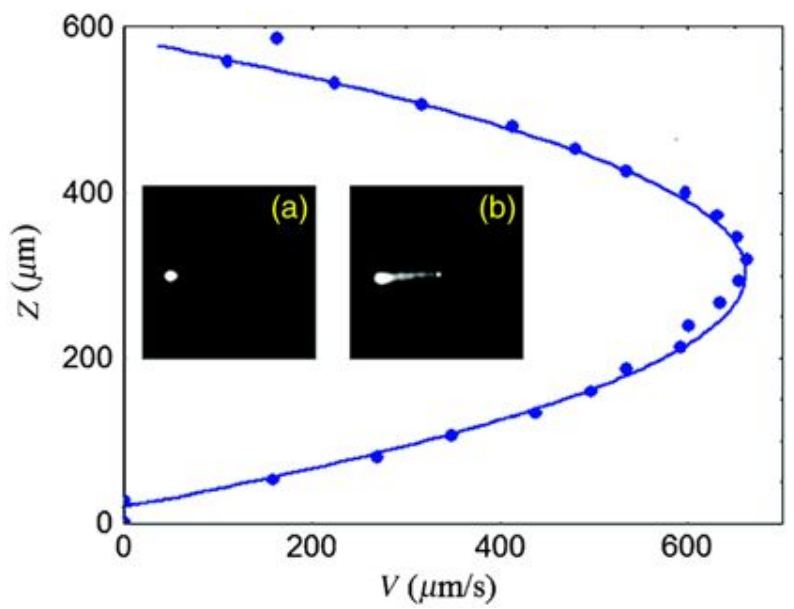

Figure 16. Results of a velocimetry experiment with a partially-coherent light source. z-scan of the longitudinal flow pattern in a straight channel, with the full line showing the predicted parabolic flow. The correlation map obtained at a depth $z=300 \mu \mathrm{m}$ in a $600 \mu \mathrm{m}$ thick channel is shown in inset (a). Inset (b) conversely shows that the correlation map obtained in a $30 \mu \mathrm{m}$ thick channel displays a "comet" effect similar to what is observed in velocimetry methods using a laser source such as HNFS. Reprinted figure with permission from Ref. [92]. Copyright (2013) by the American Physical Society.

from it the frequency dependent viscoelastic moduli [96, 95]. By contrast with traditional rheology, it requires a few microliters of material, it probes the local structure of the material and it can access higher frequencies, provided that a fast detector is used. There are in general two ways of performing microrheology experiments that are based on video particle tracking and light scattering. Once again, DFM methods can just fit in-between these two well established methodologies. In particular, we believe that fluorescence-based DFM is the ideal choice for the study of materials, whose strong scattering could mask or distort the signal from the tracer particles. Indeed, FCS has been already used with profit to study colloidal self diffusion in complex environment in strongly scattering samples [97] and we foresee a great potential for DFM that can probe collective diffusion in similar conditions.

Spatially and temporally heterogeneous systems Heterogeneity, namely the coexistence of multiple characteristic length and time scales and multiple level of organisation within the same system, is a distinctive feature of many soft and biological materials, prototypical examples being living cells and tissues. The investigation of the structural properties and the dynamics of an heterogeneous system is a very challenging task as it requires simultaneously high spatial resolution, statistical power and the access to a wide range of timescales. The idea that a suitable combination of light scattering and microscopy, complementing the averaging power of the first one the with the visual control and the spatial selectivity of the second one, could be an ideal tool for this purpose has been proposed in a variety of forms $[98,99,100]$. In all cases a laser source was used and an optical microscope was suitably customised to accommodate a traditional light scattering far field detection of the light scattered by the sample.

By contrast with the previously cited methods, DFM intrinsically offers the possibility of 
performing a space resolved study of the sample with a natural trade-off between spatial and spectral resolution. On one side, the observation length scale can be easily tuned for example by varying the objective magnification, by restricting the analysis to specific regions of interest within the field of view [50] or, on the contrary, by merging images collected in different positions or in different planes [30]. On the other hand, the same factors influence the accessible $q$-range. Increasing the magnification gives access to larger wave-vectors and using a larger image gives access to smaller wave-vectors. An elementary example of how DFM could provide a space- and q-resolved information is the following. Images of one portion of the sample can be easily partitioned in subregions and on each of them the Fourier analysis can be performed in parallel, thus obtaining a space resolved characterisation of the sample properties with a resolution given by the size of the sub-regions. A reasonable estimate for the smaller statistically relevant sub-region could be a $8 \times 8$ pixel square. By considering that the highest attainable resolution with optical microscopy is of the order of $0.2 \mu \mathrm{m}$ and by assuming a slight oversampling by a factor of two, the best final resolution of space resolved DFM is in the sub-micron range. DFM appears thus a suitable candidate for obtaining a high quality space- and q-resolved study of heterogeneous systems. We add that the implementation of imaging processing schemes based on hybrid space-frequency representations like the wavelet transform [101], could also be a viable means for the quantitative investigation of spatially heterogeneous systems [16].

As far as temporal heterogeneity i.e. non-stationarity of soft materials is concerned it would be extremely interesting to use DFM in combination with more complex forms of temporal correlations that have been proposed in the literature. Typical examples are the two-time correlation function [102] or the time resolved correlation [103] that are suitable to capture changes in the dynamics of the system.

Extracting structural information of soft materials Even though we have chosen to describe only applications devoted to the characterisation of dynamical properties, it is actually possible to use DFM for the full characterisation of the correlation properties of the sample, including the information encoded in the static structure factor $S(q)$ and in the form factor $P(q)$. In general, this entails the detailed knowledge of the transfer function $K$ for the specific imaging process. Indeed, while in general the study of the lifetime of density fluctuations is not influenced by the details of the imaging process, the extraction of the spatial Fourier power spectrum of the density fluctuation from the images is heavily based on them. We stress that beside the bare properties of the optical system such as for instance the numerical aperture and spectral distribution of the illumination beam, or the numerical aperture and aberrations of the collection optics, the transfer function $K$ is affected by a number of sample-dependent factors: scattering properties (e.g. if the sample behaves like an amplitude and/or a phase object), average refractive index, thickness, position with respect to the objective focal plane. All these elements need to be carefully accounted for when devising a strategy for the determination of $K$.

The simplest approach, which is commonly used also in traditional scattering, involves the calibration of the optical system by making use of a well characterised reference sample. An example along this line is described in Ref. [68], where the transfer function of a shadowgraph instrument was determined by making use of a dilute colloidal suspension of non-interacting nanoparticles for which both the structure factor and the form factor were substantially constant in the relevant $q$-range and the combined effect of the particle size and refractive index could be modelled theoretically. A similar approach has been demonstrated in Ref. [41], where the static structure factor of dense suspensions of colloidal hard spheres was measured in a confocal differential dynamic microscopy 
experiment. In that case, by characterising the same system in a very dilute regime, the combined effect of the optical transfer function and the form factor of the particles was characterised and used for treating the data at larger concentration.

We believe that the combined use of DFM for obtaining both dynamic and structural information could really pave the way to the routine use of optical microscopes as powerful alternatives to dynamic and static light scattering instruments. In this respect, it must be added that heterodyne DFM gives direct access to the absolute differential scattering cross section of the sample, as recently demonstrated in Refs. [28] and [50]. Indeed, for heterodyne detection, both the average image intensity and the amplitude of the signal associated to scatterers are proportional to transmitted light intensity, which allows for a self-consistent normalisation of fluctuating part of the image.

Multiply scattering systems Scattering-based DFM is ideal for applications to samples for which multiple scattering is a limiting factor. Indeed, the thickness of the samples for microscopy studies can be kept extremely small, down to a few tens of $\mu m$, which limits the amount of multiple scattering from the sample with respect to millimetre sized cells typical of light scattering. In addition, DFM with partially-coherent illumination has shown a remarkable advantage in terms of insensitivity to presence of multiple scattering [29, 92] with respect to measurements performed (both in the near and in the far field) under similar condition by using a laser illumination. Such insensitivity comes as a consequence of the limited spatial and temporal coherence of the illuminating beam that acts in reducing the effective depth of field i.e. the axial extent of the region effectively contributing to the image formation [34]. To some extent there is an analogy with previous interferometric far-field experiments (see Ref. [104] for a review) in which the combination of an interferometric setup and of a partially-coherent light source allowed quantifying the Brownian motion of colloidal particles in highly scattering media. A thorough study of this analogy and of the possible differences is needed for reaching a precise assessment of the potential of the DFM approach for the study of opaque samples such as milk and other diary products.

DFM with non-LSI imaging As pointed out in paragraph 2.4.3, whenever the imaging mechanism does not produce a linear relationship between the image intensity and the density, most of the considerations presented here are not immediately applicable unless, as we have shown above, the sample is very dilute. An important example where dilution is not possible and a novel strategy must be devised is provided by locally anisotropic molecular systems like liquid crystals (or concentrated suspensions of rod-like particles) that, when observed between crossed polarisers, show striking maps of the sample birefringence at the expense of the transmitted beam $[8,9]$. This corresponds to a homodyne detection scheme, where the intensity distribution is proportional to the square of the scattered field, which obviously breaks the linear relation between the intensity and the density. One of the possible strategies to perform quantitative DFM experiments in this condition was originally pursued in one of the seminal articles on near field scattering [15] but it was later abandoned in favour of the heterodyne scheme that proved to be superior in many respects. Nevertheless, there are cases for which it is not possible to drop the homodyne scheme and an investigation of the possibilities of DFM method in this regime would be beneficial.

Computational challenges One of the most critical aspects of DFM, especially for beginners, is data processing. The processing of DFM data can be roughly divided in two main steps: a) computation of the Fourier domain correlations between the images; b) fitting of the correlation functions with 
a suitable model. The first step involves the manipulation of large stacks of images and is is the most time and memory consuming. For this reason it also constitutes a bottleneck for the whole analysis. In this respect, a number of interesting solutions have been already proposed, which are based on the development of optimised GPU (Graphics Processing Unit)-based software [41, 56]. The resulting optimised software was capable of reducing to a few minutes the computational time needed to complete the first processing step for a typical DFM experiment. Estimates based on our experience confirm that, by suitable modifications of the existing algorithms, it should be already possible to realise a true real-time processing platform for the computation of the Fourier domain correlations in DFM experiments, with obvious advantages for the final users.

The second step, namely the fitting and the interpretation of the correlation functions, is strongly system-dependent and can benefit from the huge portfolio of analytical, numerical and computational tools that have been developed in the last decades for dynamic and static light scattering applications [3]. We believe that the full exploitation of the analogy between the DFM and light scattering, with the systematic application of the several already available tools - such as for example cumulant expansion, CONTIN algorithm, maximum entropy methods - could be of great importance in widening the field of applicability and in improving the quality of the information that can be extracted from the experimental DFM data, bringing the method at the level of the most advanced commercial light scattering instruments.

\section{Acknowledgments}

We thank S. Crotti and R. Bolis for the data in Figs. 3 and 11, respectively. We thank all the colleagues who granted permission to reproduce their published figures. We acknowledge funding by the Italian Ministry of Education and Research, "Futuro in Ricerca" Project ANISOFT (RBFR125H0M).

\section{References}

[1] Jones R A L 2002 Soft Condensed Matter (New York: Oxford University Press)

[2] Borsali R and Pecora R 2008 Soft Matter: Scattering, Imaging and Manipulation (Berlin: Springer)

[3] Xu R 2001 Particle Characterization: Light Scattering Methods, Vol. 13 (New York: Kluwer)

[4] Berne B J and Pecora R 2000 Dynamic Light Scattering: With Applications to Chemistry, Biology, and Physics (New York: Dover)

[5] Weeks ER 2010 Microscopy of soft materials in Experimental and Computational Methods in Soft Condensed Matter ed Olafsen J S (Cambridge: Cambridge University Press) pp 1-24

[6] Lu P J 2005 Confocal Scanning Optical Microscopy and Nanotechnology in Handbook of Microscopy for Nanotechnology ed Nan Y and Zhonglin (New York: Kluwer) pp 3-24

[7] Imae T, Kanaya T, Furusaka M and Torikai N (eds.) 2011 Neutrons in Soft Matter (Hoboken: Wiley)

[8] Oswald P and Pieranski P 2005 Nematic and Cholesteric Liquid Crystals: Concepts and Physical Properties Illustrated by Experiments (New York: CRC Press)

[9] De Gennes P G and Prost J 1995 The Physics of Liquid Crystals (New York: Oxford University Press)

[10] Scheffold F and Cerbino R 2007 Curr. Op. Coll. Int. Science 1250

[11] Zanchetta G and Cerbino R 2010 J. Phys.: Condens. Matter 22323102

[12] Ferri F 1997 Rev. Sci Instrum 682265

[13] Cipelletti L and Weitz DA 1999 Rev. Sci. Instrum. 703214

[14] Brogioli D, Vailati A and Giglio M 2002 Appl. Phys. Lett. 814109

[15] Giglio M, Carpineti M and Vailati A 2000 Phys. Rev. Lett. 851416

[16] Cerbino R and Vailati A 2009 Curr. Op. Coll. Int. Science 14416

[17] Goodman J W 2007 Speckle Phenomena in Optics (Englewood: Roberts \& Company)

[18] Cerbino R 2007 Phys. Rev. A 75053815

[19] Gatti A, Magatti D and Ferri F Phys. Rev. A 78063806 
[20] Magatti D, Gatti A and Ferri F Phys. Rev. A 79053831

[21] Wu M, Ahlers G and Cannell D S 1995 Phys. Rev. Lett. 751743

[22] de Bruyn J R, Bodenschatz E, Morris S W, Trainoff S P, Hu Y, Cannell D S and Ahlers G 1996 Rev. Sci. Instrum. 672043

[23] Ferri F, Magatti D, Pescini D, Potenza M A C and Giglio M 2004 Phys. Rev. E 7041405

[24] Croccolo F, Brogioli D, Vailati A, Giglio M and Cannell D S 2006 Appl. Opt. 452166

[25] Alaimo M, Magatti D, Ferri F and Potenza M A C 2006 Appl. Phys. Lett. 88191101

[26] Croccolo F, Brogioli D, Vailati A, Giglio M and Cannell D S 2007 Phys. Rev. E 76, 41112

[27] Magatti D, Alaimo M, Potenza M A C and Ferri F 2008 Appl. Phys. Lett. 92241101

[28] Cerbino R, Peverini L, Potenza M A C, Robert A, Bösecke P and Giglio M 2008 Nature Phys. 4238

[29] Cerbino R and Trappe V 2008 Phys. Rev. Lett. 100188102

[30] Ding H, Wang Z, Nguyen F, Boppart S A and Popescu G 2008 Phys. Rev. Lett. 101238102

[31] Elson E L, 2011 Biophys J. 1012855

[32] Kolin D L and Wiseman P W 2007 Cell Biochem. Biophys. 49141

[33] Kolin D L, Ronis D and Wiseman P W 2006 Biophys. J. 913061

[34] Giavazzi F, Brogioli D, Trappe V, Bellini T and Cerbino R 2009 Phys. Rev. E 80031403

[35] Rasenat S, Hartung G, Winkler B L and Rehberg I 1989 Exp. Fluids 7412

[36] Duri A, Sessoms D A, Trappe V and Cipelletti L 2009 Phys. Rev. Lett. 102085702

[37] Zakharov P and Scheffold F 2010 Soft Materials 8, 102

[38] Crocker J C and Grier D G 1996 J. Colloid. Interface Sci. 179298

[39] Kerker M 1969 The Scattering of Light and other Electromagnetic Radiation, (New York: Academic Press)

[40] Goodman J W 2005 Introduction to Fourier Optics, 3rd ed. (Englewood: Roberts \& Company)

[41] Lu P J, Giavazzi F, Angelini T E, Zaccarelli E, Jargstorff F, Schofield A B, Wilking J N, Romanowsky M B, Weitz D A and Cerbino R 2012 Phys. Rev. Lett. 108218103

[42] Brogioli D, Vailati A and Giglio M 2003 Europhys. Lett. 63220

[43] Trainoff S and Cannell D S 2002 Phys. Fluids 141340

[44] Streibl N 1985 J. Opt. Soc. Am. A 2121

[45] Nemoto I 1988 J. Opt. Soc. Am. A 51848

[46] Shibuya M, Ooki H, Saito K and Nakadate S 2005 Opt. Rev. 12105

[47] Axelrod D 2001 Traffic 2764

[48] Durisic N, Bachir A I, Kolin D L, Hebert B, Lagerholm B C, Grutter P, Wiseman P W 2007 Biophys. J. 93 1338

[49] Brogioli D, Salerno D, Cassina V, Sacanna S, Philipse A P, Croccolo F and Mantegazza F 2009 Opt. Exp. 17 1222

[50] Giavazzi F, Crotti S, Speciale A, Serra F, Zanchetta G, Trappe V, Buscaglia M, Bellini T and Cerbino R 2014 Soft Matter DOI: 10.1039/C4SM00145A

[51] Brogioli D, Croccolo F, Cassina V, Salerno D and Mantegazza F 2008 Opt. Exp. 1620272

[52] Croccolo F, Brogioli D, Vailati A, Giglio M and Cannell D S 2006 Ann. N. Y. Acad. Sci. 1077365

[53] Schatzel K 1983 Opt. Acta 30155

[54] Schulz-DuBois E O and Rehberg I 1981 Appl. Phys. 24323

[55] Krishnatreya B J, Colen-Landy A, Hasebe P, Bell B A, Jones J R, Sunda-Meya A and Grier D G 2014 Am. J. Phys. 8223

[56] Cerchiari G, Croccolo F, Cardinaux F and Scheffold F 2012 Rev. Sci. Instrum. 83106101

[57] Orihara H, Sato Y, Nagaya T, Ishibashi Y 1998 Jpn. J. Appl. Phys. 672565

[58] Orihara H, Sakai A and Nagaya T 2001 Mol. Cryst. Liq. Cryst. 366143

[59] Ortiz de Zarate J M and Sengers J V 2006 Hydrodynamic Fluctuations in Fluids and Fluid Mixtures (Amsterdam: Elsevier)

[60] Degiorgio V 1978 Phys. Rev. Lett. 411293

[61] Quentin G and Rehberg I 1995 Phys. Rev. Lett. 741578

[62] Oh J, Ortiz de Zárate J M, Sengers J V and Ahlers G 2004 Phys. Rev. E 6921106

[63] Giavazzi F and Vailati A 2009 Phys. Rev. E 80 015303(R)

[64] Vailati A and Giglio M 1997 Nature 390262

[65] Brogioli D, Vailati A, and Giglio M 2010 Phys. Rev. E 61 R1

[66] Croccolo F, Brogioli D, Vailati A, Giglio M and Cannell D S 2007 Phys. Review E 7641112

[67] Vailati A, Cerbino R, Mazzoni S, Giglio M, Nikolaenko G, Takacs C J, Cannell D S, Meyer W V and Smart A E 2006 App. Opt. 452155

[68] Vailati A, Cerbino R, Mazzoni S, Takacs C J, Cannell D S and Giglio M 2011 Nature Commun. 2290

[69] Snell E H and Helliwell J R 2005 Rep. Prog. Phys. 68799 
[70] He K, Spannuth M, Conrad J C and Krishnamoorti R 2012 Soft Matter 811933

[71] He K, Babaye Khorasani F, Retterer S T, Thomas D K, Conrad J C and Krishnamoorti R 2013 ACS Nano 7 5122

[72] Petsev D N and Denkov N D 1991 J. Coll. Int. Sci. 149329

[73] Bandyopadhyay R, Gittings A S, Suh S S, Dixon P K and Durian D J 2005 Rev. Sci. Instrum. 76093110

[74] Reufer M, Martinez V A, Schurtenberger P and Poon W C K 2012 Langmuir 284618

[75] Ferri F, D'Angelo A, Lee M, Lotti A, Pigazzini M C, Singh K and Cerbino R 2011 Eur. Phys. J. Spec. Top. 199139

[76] Mazzoni S, Potenza M A C, Alaimo M D, Veen S J, Dielissen M, Leussink E, Dewandel J-L, Minster O, Kufner E, Wegdam G and Schall P 2013 Rev. Sci. Instrum. 84043704

[77] Wilson L G, Martinez V A, Schwarz-Linek J, Tailleur J, Bryant G, Pusey P N and Poon W C K 2011 Phys. Rev. Lett. 106018101.

[78] Martinez V A, Besseling R, Croze O A, Tailleur J, Reufer M, Schwarz-Linek J, Wilson L G, Bees M A and Poon W C K 2012 Biophys. J. 1031637

[79] Jepson A, Martinez V A, Schwarz-Linek J, Morozov A and Poon W C K 2013 Phys. Rev. E 88 041002(R)

[80] Maggi C, Lepore A, Solari J, Rizzo A and Di Leonardo R 2013 Soft Matter 910885

[81] Reufer M, Besseling R, Schwarz-Linek J, Martinez V A, Morozov A N, Arlt J, Trubitsyn D, Ward F B and Poon W C K 2014 Biophys. J. 10637

[82] Paoluzzi M, Di Leonardo R and Angelani L 2013 J. Phys.: Condens. Matter 25415102

[83] Cipelletti L, Ramos L, Manley S, Pitard E, Weitz D A, Pashkovski E E and Johansson M 2003 Faraday Discuss. 123237

[84] Martens K, Angelani L, Di Leonardo R and Bocquet 2012 Eur. Phys. J. E 3584

[85] Angelini T E, Hannezo E, Trepat X, Marquez M, Fredberg J J and Weitz D A 2011 Proc. Natl. Acad. Sci. 108 4717

[86] Ding H, Millet L J, Gillette M U and Popescu G 2010 Biomed. Opt. Exp. 1260

[87] Wang R, Lei L, Wang P, Levine A, and Popescu P 2012 Phys. Rev. Lett. 109188104

[88] Potenza M A C, Alaimo M, Pescini D, Magatti D, Ferri F and Giglio M 2006 Opt. Laser Eng. 44722

[89] Alaimo M, Potenza M A C, Magatti D and Ferri F 2007 J. Appl. Phys. 10273113

[90] Magatti D, Alaimo M, Potenza M A C and Ferri F 2008 Appl. Phys. Lett. 92241101

[91] Dienerowitz M, Lee M, Gibson G. and Padgett M 2013 Lab Chip 132359

[92] Buzzaccaro S, Secchi E and Piazza R 2013 Phys. Rev. Lett. 111048101

[93] Raffel M, Willert C, and Kompenhans J 1998 Particle Image Velocimetry, A Practical Guide (Berlin: Springer)

[94] Dudderar T D, Meynart R and Simpkins P G 1998 Opt. Laser Eng. 9163

[95] Mason T G and Weitz D A 1995 Phys. Rev. Lett. 741250

[96] Cicuta P and Donald A M 2007 Soft Matter 31449

[97] Koynov K and Butt H-J 2012 Curr. Opin. Coll. Int. Sci. 17377

[98] Kaplan P D, Trappe V and Weitz D A 1999 Appl. Opt. 384151

[99] Dzakpasu R and Axelrod D 2004 Biophys. J. 871279

[100] Hiroi T and Shibayama M 2013 Opt. Express 2120260

[101] Sornette D 2004 Critical phenomena in natural sciences (2nd edn.) (Berlin:Springer)

[102] Sutton M, Laaziri K, Livet F and Bley F 2003 Opt. Express 112268

[103] Cipelletti L, Bissig H, Trappe V, Ballesta P and Mazoyer S 2003 J. Phys.: Condens. Matter 15 S257

[104] Popescu G and Dogariu A 2005 Eur. Phys. J. Appl. Phys. 3273 